\title{
Profession, Professionalität, Professionalisierung ${ }^{\prime}$
}

\author{
Axel Schmidt
}

\section{Einleitung}

Ein bedeutsames Moment gesellschaftlicher Selbsterhaltung besteht darin, dass zentrale Probleme (im Sinne einer Grundvoraussetzung für das (Weiter-)Existieren von Gesellschaften) stabilen, dauer- und musterhaften Lösungen (Institutionalisierung) zugeführt werden. Eines der Hauptprobleme von Gesellschaften besteht in der Aufrechterhaltung ihrer Strukturen trotz stetigem Wandel, welchem sie aufgrund des Wechsels der Generationen (,Personalfluktuation') unterworfen ist. Eine ,Lösung dieser Problematik ist zunächst die Differenzierung von Altersrollen, die die Einteilung in Vermittler und Empfänger des sozialen Erbes gewährleisten (Eltern-Kind-Beziehung bzw. Erzieher-Zöglings-Verhältnis). Im Unterschied zu ,naturwüchsigen` Sozialisationsprozessen (etwa innerhalb der Familie) sind Gesellschaften mit zunehmender Komplexität auf Tradierungs- (Weitergabe relevanten Wissens) und Erziehungsprozesse (Einübung in relevante Verhaltensregeln/Normenvermittlung) angewiesen, welche geplante, bewusste und zielgerichtete Handlungen zur Veränderung von Personen implizieren. ${ }^{2}$ Im Zuge der Ausdifferenzierung von Gesellschaften werden solche bedeutsamen Aspekte (etwa: Wissensvermittlung) zentraler Bereiche der gesellschaftlichen Selbsterhaltung (wie Erziehung) mehr und mehr spezialisierten Institutionen (etwa Schule) mit speziell ausgebildetem, daher in besonderer Weise qualifiziertem und berufsförmig organisiertem Personal (etwa Lehrer ${ }^{3}$ ) übertragen. Ähnliches lässt sich für die Bereiche der Medizin (Gesundheit, Heilung, Arzt, Krankenhaus), der Religion (Seelenheil, Seelsorge, Geistlicher, Kirche) und des Rechts (Gerechtigkeit Rechtssicherheit, Anwalt, Gericht) feststellen (s. hierzu ausführlich Abschn. 1.1).

Für den Bereich der Erziehung bedeutet das, dass in Abgrenzung zur ,naturwüchsigen“ (intimen, familialen, lebensweltlichen, primären) Sozialisation (innerhalb derer Erziehung

1 Dieser Artikel entstand im Rahmen eines an der Universität Frankfurt/M. begonnenen, im Rahmen der Lehramtsausbildung an der Universität Koblenz/Landau vertieften und schlieBlich an der Universität Basel fortgeführten Diskussions-, Arbeits- und Lehrkontexts. Ich danke in diesem Zusammenhang insbesondere Prof. Dr Klaus Neumann-Braun sowie Prof. Dr. Amulf Deppermann für wertvolle Hinweise.

2 Erzieherisches Handeln kann Luhmann (1995) zufolge zunächst recht allgemein als Änderung von Personen durch darauf spezialisierte Kommunikation begriffen werden. Unter Erziehung sind die psychischen Auswirkungen solcher spezifisch arrangierten Kommunikationen zu verstehen, welche absichtlich herbeigefuhrt werden und als Verbesserungen gemeint sind.

3 Die weibliche und männliche Form wechselt zufällig. 
gewissermaßen nebenbei bzw. neben vielem anderen verläuft) ein Erziehungssystem mit entsprechenden Einrichtungen (Personal, Rollen, Institutionen etc.) entsteht, dessen Leistung (zunächst) darin besteht, den Tatbestand der Erziehung herauszuheben. Eine praktische Situation (etwa Schulunterricht) in erster Linie als ,erzieherisch/pädagogisch' zu definieren (vor allem anderen was noch abläuft oder ablaufen könnte), bedeutet eine vormals offen (,diffus ') gerahmte Situation in eine spezifisch gerahmte zu überführen, nämlich in eine Situation, in der primär ,erzogen ' bzw. ,pädagogisch gehandelt' werden soll. Der Fokus liegt damit auf der systematischen Gestaltung von Bedingungen zur Erwirkung oder Verhinderung von personalen Veränderungen, zunächst ungeachtet der Frage, wer auf der Grundlage welcher Begründungen (Normativitätsproblem) die Richtung der Veränderung bestimmt (Zielkompetenz) bzw. wie solche Richtungsvorgaben zustande kommen. Solche ,sekundären Erziehungsprozesse' können als besonders bedeutsames gesellschaftliches Anliegen gelten, weshalb sie mit Hilfe gesonderten Wissens abgestützt und an entsprechend geschultes Personal delegiert werden. Kurz: bestimmte Formen des Erziehens sind für moderne Gesellschaften so zentral, dass sie nicht mehr erzieherischen Laien überlassen werden (können), sondern in die Hände von ,Erziehungsexperten' gelegt werden, welche diese Leistungen exklusiv erbringen. ${ }^{4}$ Erzieherisches bzw. pädagogisches Handeln ${ }^{5}$ unterliegt im Zuge dessen einem Prozess der Wissenssystematisierung sowie der Verberuflichung:

- Wissenssystematisierung: berufsförmig organisiertes, pädagogisches Handeln stützt sich zunehmend auf systematische Wissensformen, d.h. Wissen, welches kontinuierlich für einen bestimmten Bereich geordnet zusammengetragen und in formalisierten Ausbildungsprozessen vermittelt wird, um das Handeln in der Berufspraxis anzuleiten und es dadurch vom einfachen Alltagswissen und -handeln (der Laien, der ,naturwüchsigen' Erzieher) abhebt. Hier liegt die Wurzel des sog. Theorie-Praxis-Problems, welches als das Kernproblem der Professionalisierungsdebatte (insbesondere innerhalb der Erziehungswissenschaft) begriffen werden kann. ${ }^{6}$

- Verberuflichung: Bereits die Unterscheidung ,Beruf/Nicht-Beruf' hat für das Verständnis pädagogischen Handelns bedeutsame Folgen. Sobald Erziehung berufsförmig (,sekundär') erfolgt, unterliegt sie allgemeinen (etwa Rollenförmigkeit, Eingebundenheit in ökonomischen Austausch (etwa Arbeitsmarkt) etc.) sowie bestimmten Handlungsbedingungen (z.B. ganze Gruppen erzieherisch ,erfassen' zu müssen) sowie sich daraus ergebenden, allgemeinen (etwa emotionales Engagements dosieren zu müssen) und besonderen Handlungsanforderungen (z.B. Gruppen ,handhaben' zu können). Im Vergleich zu primärsozialisatorischen Kontexten (Familie) spezifizieren (verengen) solche Umstände

4 Nur die staatlich lizenzierte Lehrerin hat das Recht, die Resultate von Wissensvermittlungsprozessen in biographierelevanter Weise (Berufschancen) in Form gesellschaftlich anerkannter Qualifikationsindices (Zensuren, Zertifikate) zu bewerten (Prüfungsberechtigung).

5 Letztlich verbirgt sich hinter dieser begrifflichen Unterscheidung die Frage, ob und inwieweit pädagogisches Handeln (immer auch) erzieherische Ansprilche (der Veränderung von Personen. Da dieses Problem hier nicht zu lösen ist und sich die professionalisierungstheoretische Debatte gröBtenteils implizit oder explizit am erzieherischen (und damit am normsetzenden) Anspruch der Pädagogik (als einer Praxiswissenschaft) orientiert, werden die beiden Begriffe in der Folge synonym verwandt und - je nach Kontext und Aussagerichtung - mit dem Zusatz „,berufsförmig“ bzw. „professionell/professionalisiert“ versehen, wenn pädagogisches/erzieherisches Handeln im Rahmen eines Berufes organisiert ist und als Beruf ausgeübt wird.

$6 \mathrm{Vgl}$. insbesondere Dewe/Ferchhoff/Radtke (1992). 
das erzieherische Handeln (es wird rollenförmig; Lehrerrolle), die erzieherische Situation (sie ist nun eingebunden in Organisationen und damit deren formalen Abläufen und Regelungen unterworfen) und die erzieherische Beziehung (als Verhältnis zwischen Rollenträgern: Lehrer - Schüler, Sozialarbeiterin - Klientin etc.).

Berufsförmiges, erzieherisches Handeln in modernen Gesellschaften ist auf diese Weise aus Prozessen sozialer Differenzierung hervorgegangen und ist heute in wesentlicher Hinsicht durch diese geprägt, d.h. es vollzieht sich im Spannungsfeld der Trennung sozialer Sphären sowie daran geknüpfter Wissens- und Handlungsformen. Hervorgehoben seien an dieser Stelle die folgenden Aspekte:

- Die Ausdifferenzierung des gesellschaftlichen Wissensvorrates und dessen Modi der Hervorbringung, Vermittlung und Anwendung:

- Alltagswissen (Allgemeinwissen) versus Expertenwissen (Sonderwissensbereiche; Theorie) $)^{7}$

- Trennung von Wissenshervorbringung (Teilsystem ,Wissenschaft'), Wissensvermittlung (Teilsystem ,Erziehung') und Wissensanwendung (Beruf; Praxis).

- Die Entstehung eines Erziehungssystems und entsprechender Komponenten (im Zuge der Ausdifferenzierung funktionaler Teilsysteme):

- ,Naturwüchsige“ versus berufsförmig organisierte Erziehung

- spezifische Funktionsrollen (Lehrerin) versus ,diffuse‘ (Komplementär-/Inklusions-)Rollen (Schülerin, Eltern).

- Die durch Rationalisierungs- und Bürokratisierungsprozesse bedingte Entstehung formaler Organisationen mit entsprechenden bürokratischen Strukturen und rationalen Zielsetzungen (pädagogische Einrichtungen wie Schulen) auf der einen versus die ,Welt ' der Adressaten (Lebenswelt, Familie) auf der anderen Seite.

Das Auseinandertreten von Alltags- und Expertenwissen, von Wissenschaft und Berufspraxis, von Ausbildungswissen und praktischem Erfahrungswissen, von naturwüchsiger und berufsförmiger Erziehung sowie der Sphären formaler (pädagogischer) Organisationen sowie deren Personal auf der einen und der (intimen, informalen) Lebenswelt der Adressaten solcher Institutionen auf der anderen Seite bringt in der Moderne die Notwendigkeit eines Berufstypus hervor, welcher zwischen den auf diese Weise ,getrennten Welten'vermittelt, indem er die entstandenen Distanzen interagierend überbrückt: der/die Professionelle. Das Konzept der Profession(-alisierung/-alität) lässt sich somit in sehr allgemeiner Weise zunächst innerhalb eines spezifischen Problemkontextes verorten, nämlich dem Aufbrechen von Differenzen und der Entstehung von Distanzen in der Moderne, sowie innerhalb der darauf bezogenen strukturellen Lösungen, nämlich der Tätigkeit des Vermittelns und Überbrückens entstandener Differenzen und Distanzen durch einen spezifischen Berufstypus.

Historisch betrachtet, lassen sich die Professionen damit zunächst als gesellschaftliche 'Einrichtungen' (Institutionen) fassen, welche im Übergang von Vormoderne zu Moderne als ,Reaktionen' auf spezifische ,Anfälligkeiten' der modernen Gesellschaft (etwa Anonymisierung, Wissensdifferenzierung, Komplexitätssteigerung etc.) entstanden sind und aufgrund ihres Potenzials zur Lösung spezifisch mit der Moderne verbundener Probleme zu herausge-

7 Vgl. Pfadenhauer (2003: 23 ff.). 
hobenen, berufsförmig organisierten und für die moderne Gesellschaft besonders relevanten Tätigkeitskomplexen verdichtet wurden. ${ }^{8}$ Das wissenschaftliche und öffentliche Interesse an den neuentstandenen Professionen spiegelt sich in Debatten und Diskursen um das Phänomen der Professionalität/-alisierung, insbesondere dann, wenn - wie im Falle pädagogischer Professionalität - strittig ist, ob es sich um (Tätigkeits-)Bereiche handelt, die professionell, professionalisierungsbedürftig oder überhaupt professionalisierbar sind. ${ }^{9}$ In synchroner, heutiger Perspektive sind Professionen herausgehobene Berufsformen, die bestimmte gesamtgesellschaftlich zentrale Funktionen erfüllen und die sich aufgrund dessen anhand gemeinsamer typischer Merkmale charakterisieren lassen (Abschn. 1.1). Mit Blick auf das Handeln der Professionellen lassen sich Professionen als Interaktionskomplexe begreifen, denen aufgrund ihres Eingebundenseins in strukturelle Zwänge eine eigentümliche Handlungslogik (s. Glossar) zugeschrieben werden kann (Abschn. 1.2). Anstatt das Konzept der Professionalität durch die Generalisierung typischer Merkmale der klassischen Professionen (Abschn. 1.1) auf ein idealtypisches Bild der Profession/des Professionellen festzulegen und damit (unzulässig) zu verengen, versuchen struktur- und handlungstheoretische Ansätze die innere Logik professionellen (pädagogischen) Handelns zu rekonstruieren (Abschn. 1.2). Insbesondere merkmalstheoretische und funktionalistische Idealbilder des Professionellen (Abschn. 1.1) ließen pädagogische Berufstätigkeiten im Hinblick auf Professionalität defizitär und im Hinblick Professionalisierbarkeit problematisch erscheinen (Abschn. 2.1). Lösungen und Auswege aus diesem Dilemma behandelt Abschnitt 2.2. In Abschnitt 2.3. soll versucht werden, Aspekte der Logik professionellen Handelns an pädagogischen Handlungsfeldern (vornehmlich mit Blick auf Schule sowie kontrastierend mit Blick auf Sozialarbeit/Sozialpädagogik) aufzuzeigen.

8 Zur Entstehung der klassischen Professionen vgl. Stichweh (1992 und 1996), speziell zur historischen Genese pädagogischer Berufe vgl. Apel et al. (1999), mit Fokus auf den Komplex ,Sozialarbeit/Sozialpädagogik 's. Merten/Olk (1996).

9 Einen kurzen Überblick uber den Verlauf der Professionalisienungsdebatte geben Dewe/Otto (2001) sowie Dewe/Ferchhoff/Radtke (1992). Zu Unterscheidung von faktischer Professionalisiertheit und Professionalisierungsbedurftigkeit vgl. Oevermann (1996: 135 ff.). 


\section{Professionssoziologische Konzepte}

Aus heutiger Sicht erscheint der Diskurs um (pädagogische) Professionalität vornehmlich durch zwei, sich in zeitlicher Hinsicht ablösende Theoriestränge geprägt: Nämlich zunächst durch Konzepte, die an den sog. klassischen Professionen entwickelt wurden und (ideal-)typische Merkmale zur Bestimmung von Professionalität entwickelten (Abschn. 1.1) sowie in der Folge durch theoretische Bemühungen, welche solche merkmalstheoretischen (indikatorischen) Zugänge zum Phänomen der Professionalität durch eine Fokussierung der Handlungslogik zu überwinden versuchten (Abschn. 1.2). Insbesondere die Entwicklung eines eigenständigen Konzepts pädagogischer Professionalität (Abschn. 2) lässt sich vor diesem Hintergrund als Überwindung merkmalstheoretischer (defizitärer) Ansätze (Abschn. 2.1) unter Rückgriff auf handlungs- und strukturtheoretische Zugänge (Abschn. 2.2) begreifen.

\subsection{Ausgangsbasis: Funktion und typische Merkmale klassischer Professionen}

Professionstheoretische Konzepte der 1960/70er Jahre folgten größtenteils dem strukturfunktionalistischen Paradigma. ${ }^{10}$ Um von einer Profession zu sprechen, ist demzufolge mehr erforderlich, als dass es sich um eine berufsförmig organisierte Tätigkeit handelt (oben als Verberuflichung bezeichnet), welche systematisches Wissen (oben als Wissenssystematisierung bezeichnet) zum Einsatz gelangen lässt. Das Konzept der Profession lässt sich schärfen, indem man die Übergänge von „Arbeit“" zu „Beruf“ hin zur „Profession“ auf einer Wissensund einer Sozialdimension spezifiziert:

- Wissensdimension: Steigerung der Wissenssystematisierung zur Verwissenschaftlichung (Entstehung von Theoriewissen). Zum Anspruch auf Effizienz (praktische, ,funktionierende' Problemlösungen) tritt der Anspruch auf Erklärung (der Fokus liegt nicht darauf dass, sondern warum etwas ,funktioniert'). Probleme werden nicht nur, irgendwie ' gelöst (dauerhaften, praktischen Lösungen zugeführt), sondern auch hinsichtlich fallunspezifischer Problemgründe erklärt.

- Sozialdimension: Prestigesteigenung durch besondere Wertbindung (Kollektivorientierung; s.u.).

Dieses funktionalistische Konzept der Profession dient als Grundlage, (proto-)typische Merkmale der Professionen zu formulieren (Merkmalstheorie; indikatorisch). Diese lassen sich als Beurteilungskriterien verwenden, um einzuschätzen, ob eine (empirisch beobachtbare) Berufstätigkeit als (nicht-)professionell bezeichnet werden kann bzw. wie weit sie von einer Profession im idealtypischen Sinne entfernt ist. Folgende Merkmale auf folgenden Dimensionen gelten diesem Konzept zufolge als professionstypisch: ${ }^{11}$

- Kompetenzdimension

- Rekurs auf wissenschafiliches Wissen: Professionen beziehen sich auf disziplinäre Wissenstraditionen (etwa Medizin oder Pädagogik) und deren Theoriewissensbestände.

$10 \mathrm{Vgl}$. etwa Hartmann (1972).

11 Vgl. auch Kurtz (2002: 47 ff.) sowie - verschiedene Theoriepositionen vergleichend - Pfadenhauer (2003: $31 \mathrm{ff}$.). 
- Spezifische Handlungskompetenz, die prinzipiell darin besteht, theoretisches Wissen praktisch umzusetzen. Dies soll (u.a.) dadurch gewährleistet werden, dass Professionelle nicht nur theoretisch ausgebildet werden, sondern im Rahmen ihrer Ausbildung auch gezwungen sind, theoretisch erworbenes Wissen in die Praxis einfließen zu lassen, was in Berufspraktika bzw. in praktischen Ausbildungsphasen (etwa Referendariat) geschieht.

- Spezialisierung und eigenständige Fachlichkeit: Grundlage der Professionen sind somit spezielle Wissensbestände und Handlungsformen. Hieraus resultieren fachliche Standards ${ }^{12}$, wodurch sich Professionen einerseits vom Alltag (von Laien) und andererseits untereinander unterscheiden.

- Leistungsdimension

- Zentralwertbezug: Professionen erbringen gesellschaftlich unverzichtbare Leistungen (Seelenheil, Gesundheit, Gerechtigkeit) und sind damit an zentrale (universelle) Werte der Gesellschaft (d.h., bezogen auf die Medizin etwa, dass (menschliches) Leben prinzipiell als erhaltenswert gilt) gebunden.

- Kollektivorientierung: Professionen unterliegen aufgrund ihres Zentralwertbezugs einer am Gemeinwohl ausgerichteten Handlungsorientierung (zumindest berufsständiger Ideologie zufolge). Da Professionen i.d.S. ,soziale Dienstleistungen“ erbringen, unterliegt die Orientierung an Erwerbsinteressen diversen, symbolisch bedeutsamen Vorschriften und Kontrollen (bei Ärzten etwa: Verbot von Eigenwerbung und Honorarverhandlungen, Pflicht jeden Patienten zu behandeln).

- Verwaltung , legitimen Wissens': bezüglich ausgrenzbarer sozialer Handlungsprobleme (bezogen auf die Medizin: Krankheit) verwalten Professionen ein wissenschaftsgeneriertes (daher: ,legitimes') Wissen zur Problemdeutung (med.: Diagnose) und Lösung (med.: Therapie).

- Soziale Kontrolle und Gesellschafisintegration: Funktion des Deutungs- bzw. Problemlösemonopols der Professionen ist es, Sinninterpretationen sozial zu vermitteln und damit Wertregulierungen gesellschaftlich sicherzustellen.

- Theoriewissen, anwendende'Tätigkeit: In sehr allgemeiner Hinsicht ist es damit die Hauptaufgabe oder Funktion der Professionen, relevantes, innerhalb des Wissenschaftssystems akkumuliertes (Theorie-)Wissen an ,kollektiv bedeutsamen' Stellen interagierend und fallbezogen in den Prozess gesellschaftlicher Reproduktion ,einzuspeisen".

- Strukturdimension (äußere und innere Strukturen)

- äußere (Einbettung in gesellschaftliche Strukturen)

- horizontal (Beziehung zu anderen sozialen Einheiten)

- Staatsautonomie: Professionelle sind nicht in direkter Weise staatsabhängig (wie etwa Beamte). Idealtypisch ist die berufliche Stellung als Selbständiger bzw. Freiberufler.

- Klientenautonomie: Das Aufsuchen eines Professionellen (etwa eines Arztes) obliegt der freien Entscheidung des Klienten. Umgekehrt ist der Professio-

12 Unter fachlichen Standards sind i.A. ,Methoden' und Anwendungsprozeduren der jeweiligen akademischen Berufe zu verstehen, welche dem, gesicherten Wissen' der disziplinären Bezugswissenschaften (i.S.e. ,state of the art") korrespondieren. Z.B.: medizinische Diagnoseverfahren wie die Kniearthroskopie oder Methoden der schulischen Wissensvermittlung (etwa grundschuldidaktische Konzepte wie fácherubergreifender Projektunterricht). 
nelle in seinen Entscheidungen nicht an die ,Weisungen" seines Klientel (im Sinne einer reinen Dienstleitung) gebunden.

- Auf einer solchen, doppelten Autonomie fußt die sachlich gebundene Entscheidungsautonomie des Professionellen, die darin besteht, dass eine Entscheidung (etwa welche Therapieform einem bestimmten Patienten vorgeschlagen wird) weder administrativ determiniert ist (im Sinne eines Programms) noch den Wünschen oder Bedürfnissen des Klientel untergeordnet wird (im Sinne einer reinen Dienstleistung), sondern allein auf der Grundlage des vorliegenden Sachverhalts gefällt wird (im Beispiel: Therapieform A wird vorgeschlagen, weil es die Sache (hier: die diagnostizierte Krankheit) erfordert, und nicht weil es so vorgeschrieben ist oder weil es der Patient so wünscht).

- vertikal (Prestigegefälle in Relation zu ,einfachen' Berufen)

- Staatliche Lizenzierung: Professionen ,besitzen“ die staatliche Lizenz (Berechtigung zur Berufsausübung) für die Gesellschaft sowie die Identität und/ oder Existenz eines Klienten wertvolle Dinge zu verwalten und diese zu ,versachlichen'. Diese Berechtigung ist gekoppelt an die im und durch das Examen anerkannte Qualifikation.

- Exklusivität: Professionen besitzen ein Monopol (exklusive Lizenz) für die Erbringung ,sozialer Dienstleistungen“ innerhalb eines bestimmten Bereichs. D.h.: bestimmte Tatbestände und daran geknüpfte Handlungsformen (etwa ein operativer Eingriff aufgrund einer schwerwiegenden Krankheit) lassen den Einsatz eines spezifischen Professionellen (Arzt, Chirurg) zwingend werden. Das Problem darf und kann i.d.R. auch nicht durch Laien gelöst werden.

- Hohes Prestige: Aufgrund ihrer Kollektivorientierung und ihres Exklusivanspruchs für zentralwertbezogene Bereiche genießen Professionen eine besonders hohe öffentliche Anerkennung.

- innere (interne Organisation/Strukturen)

- Berufliche Autonomie: Der Professionelle ist beruflich selbstständig, d.h. prinzipiell weder vom Staat noch vom Klientel in seinen Entscheidungen abhängig (s.o.).

- Professionen sind aufgrund dessen korporativ organisiert ${ }^{13}$, was es des weiteren mit sich bringt, dass Professionen

- sich durch die Kodifizierung berufsethischer Normen auszeichnen,

- internen Kontrollen durch Berufsverbände (z.B. Ärztekammer) unterliegen,

- demzufolge sowohl fachliche Standards der Berufsausübung als auch Standards der Ausbildung (Personalrekrutierung) selbst kontrollieren,

- einem Berufs- bzw. Standesethos (etwa ,hippokratischer Eid") verpflichtet sind, welcher als professionsinterne Verkörperung des Zentralwertbezugs zu begreifen ist,

- sich durch berufsständische Solidarität auszeichnen und

13 Hierunter ist im vorliegenden Zusammenhang eine berufsständisch-körperschaftliche Organisationsform als Zusammenschluss freiberuflich Tätiger (etwa: Ärztekammer) zu verstehen (vgl. hierzu Stichweh (1996: 65)). 
- ihr Personal durch höhere Qualifzierung (lange wissenschaftliche Spezialausbildung in formalisierten, wissenschaftlichen Ausbildungsgängen verbunden mit Praktika (Referendariat, Vikariat)) rekrutieren und damit Zugangskontrollen mittels universitärer Leistungsorientierung betreiben.

- Handlungs- und Habitusdimension

- Sachgebundener Habitus: Professionelle erwerben im Laufe ihrer Berufssozialisation eine , an der Sache' orientierte Haltung, welche sie befähigen soll, subjektive Probleme ,sachlich' (objektiv, nüchtern) zu handhaben. D.h. insbesondere auch unter Ausklammerung der jeweiligen Subjektivität der Person sowie ihrer fallunspezifischen, sonstigen Besonderheiten (etwa ihr Geschlecht, ihr Alter, ihre Hautfarbe, ihre Religionszugehörigkeit, ihr Sprachduktus etc.).

- Die Orientierung an der Sache geschieht zum Wohle des Klienten, d.h. auf dessen Betreiben hin und in dessen Interesse. Dennoch erfolgt dies nach dem Prinzip einer Vermittlung von Sachthematiken an "sachfremde" Personen (Laien), wodurch eine für die Professionellen-Klient-Beziehung konstitutive Wissensasymmetrie entsteht (eine ärztliche Behandlung geschieht zwar auf Betreiben und im Interesse eines Patienten, rekurriert jedoch auf Wissensbestände, die dem Patienten fremd sind, diesem also von einem ,Wissenden ', vermittelt' werden müssen).

- Diese Wissensasymmetrie setzt eine temporäre Vertrauensbeziehung zwischen Professionellem und Klient voraus, da der Klient nicht wissen kann, was der Professionelle weiß und aufgrund dessen auch nicht (jederzeit) kontrollieren kann, ob er zu seinem Wohle handelt. Der Klient ist also (temporär) gezwungen - möchte er hinsichtlich seiner subjektiven (Problem-)Lage von höheren Wissensformen profitieren -, den Vorschlägen des Professionellen zu vertrauen. M.a.W.: Er ist in seinem eigenen Interesse gezwungen sich temporär und aspektuell ,in Vormundschaft" zu begeben.

In Abgrenzung zur einfachen Berufsförmigkeit bezeichnet „Professionalität“ also einen Zustand, gesteigerter Berufsförmigkeit', welcher sich v.a. dadurch ergibt, dass die Berufsausübung an das längere Einsozialisieren in eine durch eine wissenschaftliche Disziplin (etwa Medizin) vertretende Wissenstradition gebunden ist, was die Grundlage dafür ist, dass der/die auf diese Weise Ausgebildete ((Fach-)Wissenserwerb) und Einsozialisierte (Erwerb eines Berufethos) die Berechtigung erwirbt (Lizenz), gesellschaftlich als zentral erachtete Tätigkeiten (etwa (Seelen-)Heilen, Recht sprechen, Lehren) am anempfohlenen Menschen (etwa Patient, Klient, Schüler) , autoritativ' zu dessen Wohle (Mandat) auszuführen. ${ }^{14}$ Professionalität wird sozialen Handlungsfeldern, (pädagogischen) Institutionen/Organisationen sowie (pädagogischen) Rollen, Personen und deren Praxis der Berufsausübung als positive Eigenschaft (professionell vs. nicht-professionell) zugeschrieben. Die Zuschreibung von

14 Während die Lizenz die formale Berechtigung umfasst, bestimmte Handlungen durchfüren zu dürfen (etwa das Verschreiben rezeptpflichtiger Medikamente), verweist das Mandat auf eine umfassendere Definitionsmacht, „nämlich die Vollmacht bzw. ,Ermächtigung“, , autoritativ“ tätig zu werden“" (Pfadenhauer 2003: 50). Hierzu gehört etwa, bestimmen zu können, unter welchen Umständen professionelle Hilfestellungen oder Eingriffe erforderlich sind und wie sie konkret gestaltet werden, d.h. worin etwa die Hilfestellung besteht. 
Professionalität fungiert v.a. dadurch als ,Prädikat', dass die Legitimation, als ausgebildeter, ,praktischer Experte' einer gesellschaftlich bedeutsamen Tätigkeit nachzugehen, öffentlich anerkannt wird, was zur Macht- und Statuserhöhung der Berufsgruppe beiträgt. Professionalität ist somit immer auch ein reform- und berufsstrategisches Konzept, welches immer auch unter machttheoretischen Gesichtspunkten betrachten lässt. ${ }^{15}$

Als klassische oder vollwertige Professionen werden nun jene Berufsgruppen bezeichnet, welchen die hier aufgeführten Merkmale vollständig zugeschrieben werden können. Sie stellen in diesem Sinne Prototypen des klassischen Professionalisierungskonzeptes dar.

\subsection{Strukturzwänge und eigentümliche Handlungslogik}

Seit den 1980er Jahren ist ein Perspektivenwechsel in der Professionalisierungsdebatte zu verzeichnen: Aktuelle Professionalisierungstheorien betonen weniger die gesellschaftliche Einbettung bzw. die sich daraus ergebenden (Struktur-)Merkmale (s.o.), sondern fokussieren die Praxis der Ausübung der Profession und damit das Handeln des Professionellen bzw. die Interaktion zwischen Professionellem und Klientin. Der Fokus verschiebt sich also von der Profession als statischer, sozialstruktureller und funktionaler Größe hin zum professionellen Handeln als der dynamischen, prozessualen und akteursgebundenen Seite von Professionalität. Die Frage ist dann weniger, durch welche Merkmale sich Professionen im Allgemeinen auszeichnen bzw. welche Wissensgrundlagen wie umgesetzt werden müssen, um für das bestehende Gesellschaftssystem funktionale Resultate zu erzielen, sondern vielmehr was professionelles Handeln faktisch ausmacht und wie Professionalität in und durch Handlungen in jeweils spezifischen Bereichen mit jeweils eigentümlichen Strukturen (re-)produziert wird. ${ }^{16}$ Aus dieser Perspektive wird professionelles Handeln als durch eine besondere Handlungslogik bestimmt gesehen, welche sich aus spezifischen Strukturbedingungen bzw. spezifischen (funktionalen) Anforderungsbedingungen ergibt, denen professionelles Handeln unterworfen ist. So lässt sich professionelles Handeln von Handlungstypen anderer Bereiche - etwa dem bürokratischen (Verwaltung), dem wirtschaftlichen (Markt) oder dem schlichtweg alltäglichen Handeln (Lebenswelt) - abgrenzen. Das besondere Profil professionellen Handelns lässt sich in Form einer professionellen Handlungslogik (kurz: ,Professionslogik') verdichten. Eine solche Professionslogik soll im Folgenden umrissen werden.

Ein sehr allgemeines Kennzeichen professioneller Handlungslogik (,Professionslogik') ist der widersprüchliche Charakter, welcher alles professionelle Handeln gleichermaßen betriffi. D.h. die Handlungsanforderungen und -ziele sind in Widersprüche (Paradoxien)

15 Vgl. Daheim (1992).

16 Professionalität über die Rekonstruktion besonderer, bereichstypischer Handlungsanforderungen bzw. -logiken zu erfassen, ist Ansätzen aus der (strukturfunktionalen) Handlungstheorie (Parsons 1965; Brunkhorst 1996), der strukturtheoretischen Soziologie (Oevermann 1996), der (Luhmann'schen) Systemtheorie (Stichweh 1992. 1996) sowie der interaktionistischen Sozialtheorie (Schütze 1992, 1996) gemeinsam. Das vorliegende Unterkapitel ist ein Versuch, Gemeinsamkeiten (bei aller Unterschiedlichkeit) dieser theoretischen Ansätze im Hinblick auf eine handlungs- bzw. strukturtheoretische Fassung von Profession(-alität, -alisierung) herauszustellen. Die folgenden Ausfuhrungen sind mithin an der Sache (Was ist Professionalität?), nicht an theoriespezifischen Differenzierungen orientiert. 
verstrickt, welche - aufgrund ihres strukturellen Charakters - nicht auflösbar, wohl aber (praktisch in unterschiedlicher Weise) bewältigbar sind. Prototypisch ist diese paradoxale Grundfigur professioneller Handlungslogik am ärztlichen Handeln festgemacht worden. ${ }^{17}$ Grundlage ist folgendes Schema allgemeiner Handlungsorientierungen (auch: Mustervariablen oder pattern variables) nach Talcott Parsons ${ }^{18 .}$

\section{Prototyp: Primärgruppe/Gemeinschaft Prototyp: Sekundärgruppe/Gesellschaft}

\begin{tabular}{|c|c|c|}
\hline 1 & Diffusität & Spezifität \\
\hline & $\begin{array}{l}\text { HO: umfassend, die ganze Person betreffend } \\
\text { H: persönliche Probleme besprechen } \\
\text { B: Familie, Freundschaft, Partnerschaft }\end{array}$ & $\begin{array}{l}\text { HO: einzelne Aspekte betreffend, rollenförmig } \\
\text { H: Einkaufen, Amtsgang } \\
\text { B: Käufer - Verkäufer, Beamter - Leistungs- } \\
\text { empfänger }\end{array}$ \\
\hline \multirow[t]{2}{*}{2} & Partikularität & Universalität \\
\hline & $\begin{array}{l}\text { HO: Orientierung an gruppenspezifischen } \\
\text { Normen } \\
\text { H: BegrüBungsrituale in Subkulturen } \\
\text { B: Gruppen, Milieus, ,kleine Lebenswelten“ }\end{array}$ & $\begin{array}{l}\text { HO: Orientierung an gesamt- } \\
\text { gesellschaftlichen Normen } \\
\text { H: Vertragsabschluss, Wählen, Arztgang } \\
\text { B: Öffentlichkeit, Arbeitsbeziehungen } \\
\end{array}$ \\
\hline \multirow[t]{2}{*}{3} & Kollektivorientierung & Selbstorientierung \\
\hline & $\begin{array}{l}\text { HO: an allgemeinen Interessen orientiert } \\
\text { H: politisches Handeln } \\
\text { B: Arzt - Patient, Lehrer - Schüler }\end{array}$ & $\begin{array}{l}\text { HO: an eigenen Interessen orientiert } \\
\text { H: wirtschaftliches, karrieristisches Handeln } \\
\text { B: wirtschaftliche Tauschbeziehungen }\end{array}$ \\
\hline \multirow[t]{2}{*}{4} & Affektivität & affektive Neutralität \\
\hline & $\begin{array}{l}\text { HO: Emotionen steuem das Handeln } \\
\text { H: Streit in der Familie, ,Beziehungsprobleme' } \\
\text { B: Partnerschaft, Freundschaft, Familie }\end{array}$ & $\begin{array}{l}\text { HO: Die Sache steuert das Handeln, Emoti- } \\
\text { onen bleiben ausgeblendet } \\
\text { H: wiss. Vorträge, bürokratische Amtshand- } \\
\text { lungen } \\
\text { B: gesellschaftliche Beziehungen (Schaffner } \\
\text { - Zuggast) }\end{array}$ \\
\hline \multirow[t]{2}{*}{5} & Zuschreibung (ascription) & Leistung (achievement) \\
\hline & $\begin{array}{l}\text { HO: Status aufgrund askriptiver Merkmale } \\
\text { H: Beurteilung einer Person anhand unver- } \\
\text { änderlicher Merkmale (Alter, Geschlecht, } \\
\text { Ethnizität) } \\
\text { B: Familie, Freundschaft, Bekanntschaft }\end{array}$ & $\begin{array}{l}\text { HO: Status aufgrund erworbener Merkmale } \\
\text { H: Beurteilung einer Person anhand konkreter } \\
\text { Leistungen } \\
\text { B: Professionen (z.B. Arzt), Lern-Lehr-Bezie- } \\
\text { hungen }\end{array}$ \\
\hline
\end{tabular}

$\mathrm{HO}=$ Handlungsorientierung $\mathrm{H}$ : beispielhafte Handlung/Handlungstypus B: beispielhafte institutionalisierte Interaktions- oder Beziehungsform

Schaubild 1: Pattern Variables (mit erläuternden Beispielen)

17 Vgl. Parsons (1965).

18 Eine zusammenfassende Darstellung hinsichtlich professionalisierungstheoretischer Fragen findet sich bei Wernet (2003). 
Unter Rekurs auf diese prinzipiell möglichen Handlungsorientierungen wird Professionalität in Abgrenzung zu marktförmigen/wirtschaftlichen, administrativen/bürokratischen sowie alltäglichen/lebensweltlichen Handlungsformen als auf höherstufiger, universeller Solidarität beruhend bestimmt. Unter Zuhilfenahme der Pattern Variables dimensioniert und spezifiziert Brunkhorst (1992: 51 ff.) die Logik professionellen Handelns wie folgt:

- Leistungsorientierung (achievement) statt Zuschreibung (Attribution; ascription): an die Stelle auf Zuschreibung beruhender, traditionaler Autorität (etwa qua Stand, Charakter oder Alter) tritt die Fachautorität, welche durch Leistungen (Qualifikationen) zu erwerben ist und sich über die Logik des besseren Arguments (Sachlogik) bzw. die konsensuelle Logik theoretischer Diskurse zu legitimieren hat.

- Universalismus statt Partikularismus: an die Stelle einzelner, tradierter Ordnungen (Lebenswelt, Gemeinschaft, Kleingruppe) tritt die Allgemeingültigkeit der wissenschaftlichen Erkenntnis. Partikulare Formen (Freund-Feind-Moral, Separatismen) werden ersetzt durch ,höherstufigere' (d.h., die Grenzen der kleinen Gemeinschaft überschreitenden) Formen der Solidarität, welche dem gesamtgesellschaftlichen Wertekonsens (etwa: die Erhaltungswürdigkeit menschlichen Lebens, das Recht auf Bildung und Persönlichkeitsentwicklung etc.) korrespondieren (sollten).

- Spezifität statt Diffusität: an die Stelle ursprünglicher Ganzheitlichkeit, d.h. ,naturwüchsiger', primärer und intimer (= diffuser) Beziehungen treten funktionale (Rollen-) Spezifizierungen. Handlungen sind nicht an der ,ganzen Person' ausgerichtet, sondern fokussieren sachbezogene und verallgemeinerbare Aspekte der Person (etwa: einen Krankheitszustand oder entwicklungsstandsspezifische Wissensdefizite). Dies bedeutet umgekehrt, dass ,alles andere', was eine Person ausmacht, innerhalb spezifischer Rollenbeziehungen zunächst ausgeschlossen ist bzw. einer Begründung bedarf, um inkludiert zu werden.

- Affektive Neutralität statt Affektivität: emotionale Involviertheit weicht einer affektiven Neutralität, welche sich in Verunpersönlichung (Distanziertheit) und Versachlichung der Beziehung niederschlägt.

- Kollektivitätsorientierung statt Selbstorientierung: Entscheidendes Moment höherstufiger Solidarität ist, dass trotz der Verunpersönlichung und Versachlichung der Beziehung eine verallgemeinerte (kollektive) Wertbindung bestehen bleibt. M.a.W.: „Die Solidarität gewachsener Gemeinschaften verdampft. Was allein zurück bleibt, ist deren moralische Potenz: die ,Kollektivitätsorientierung' der Professionen...“ (ebenda: 53).

Das Konzept der höherstufigen, universellen Solidarität lässt sich anhand der obigen Aufschlüsselung zuspitzen auf den paradoxen Versuch, „Solidarität unter Fremden“ (Brunkhorst 1996) herzustellen, welche als prinzipielle Voraussetzung für ,die Arbeit am Menschen` gelten kann. ${ }^{19}$

Anlass zur Etablierung einer solchen ,Solidarität' ist die Notwendigkeit der Bewältigung einer lebenspraktischen Krise aufseiten des Klienten, welche nicht (mehr) im und durch den All-

19 Talcott Parsons betont etwa, ,dass die funktionale Bedeutung der Kombination von Universalismus, funktionaler Spezifität und emotionaler Neutralität in erster Linie darin liegt, dass diese dem Arzt erlaubt, hinreichend in die Privatsphäre oder die persönlichen Beziehungen des Patienten einzudringen, um seine Aufgabe zu erfullen. Durch eine solche Definition der Rolle wird es möglich, Widerstände zu ubberwinden oder auf ein Minimum herabzusetzen, die sonst die ärztliche Tätigkeit sehr wohl unmöglich machen könnten“" (Parsons 1965: 37). 
tag selbst gelöst werden kann und deshalb die Konsultierung eines ,Experten' (Professionellen) erfordert. M.a.W.: An dem Punkt, an dem das Ausmaß des Leidensdrucks die Fortsetzung einer autonomen Lebenspraxis verunmöglicht und die Selbstauslegungs- und Selbstheilungskraft des Alltags zur Wiederherstellung dieser erschöpft ist, wird das Wissen eines Experten zur Lösung des Problems herangezogen (prototypisch: der Gang zum Arzt). Dieser hat nun die Aufgabe (das Mandat) und qua Qualifikation die Berechtigung (Lizenz), sich dem Problem (dem Anliegen, , der Sache') des Klienten dergestalt zu widmen, dass ,diesem geholfen ist' (,zum Wohle des Klienten'). Dies geschieht prinzipiell dadurch, dass der Professionelle sein „höhersymbolisches Wissen“20, seine Deutungskompetenz zur ,Behandlung' und ,Lösung' des jeweiligen konkretes Falles einsetzt. D.h., sich der Besonderheit (Spezifik) des jeweiligen Falls widmet und diesen vor dem Hintergrund theoretischen (universelle Erklärungen bereitstellenden) Wissens (um-)deutet und damit Lösungswege aufzeigt. M.a.W.: Der Professionelle hilft dem Klienten, indem er diesen (wieder) befähigt, sich (langfristig) selbst zu helfen.

Professionen werden deshalb als institutionalisierte Handlungssysteme stellvertretender Deutung sowie als Strukturorte der verwissenschaftlichten Krisenbewältigung resp. der Vermittlung von Theorie und Praxis verstanden. Die eigentümliche Deutungsarbeit stellt dabei den Kern professioneller Tätigkeit dar und lässt sich als Dialektik von universalisierter Regelanwendung und hermeneutischem Fallbezug 21 begreifen.

Da Professionen (inter-)personale Probleme (Seelenheil, Gesundheit, Interessensausgleich, Wissenserwerb) auf der Grundlage wissenschaftlichen Wissens behandeln, d.h. Personenveränderungen durch das ,Anwenden 'von Sonderwissen betreiben, sind sie auf Interaktion mit diesen Personen angewiesen.$^{22} \mathrm{Da}$ einfache Interaktionssysteme prinzipiell höhere Scheiternsrisiken mit sich bringen als organisationale Strukturen ${ }^{23}$, wird die von der prinzipiellen Interaktionsabhängigkeit professionellen Handelns ausgehende Gefahr mittels institutionalisierter Handlungssysteme (etwa Arzt-Patient-Interaktionssystem) abgestützt. Die Beziehung zwischen Professionellem und Klient folgt auf diese Weise strukturlogisch bestimmten Mustern, sie ist - mit den Parson'schen pattern variables gesprochen - zunächst prinzipiell funktional spezifisch, universalisiert, affektiv neutral, kollektivorientiert und erfolgt auf der Basis einer Leistungsorientierung (s.o.). Kurz: Es kommt zur Ausbildung einer Rollenbeziehung, welche gefasst werden kann als asymmetrische Beziehungsform zwischen einer funktionsspezifischen Rolle (Arzt) auf der einen und einer diffusen Inklusionsrolle ( $\mathrm{Pa}$ tient) auf der anderen Seite.

Die Notwendigkeit innerhalb moderner Gesellschaften, die Bearbeitung personaler (intimer) Probleme an die stellvertretende Deutungsleistung anonymer Experten zu delegieren, erzeugt jene eigentümliche (oben angedeutete) Beziehungsstruktur (prototypisch: Arzt-Patient-Beziehung), welche sich generell zunächst dadurch auszeichnet, dass sie in spezifische Spannungen und Widersprüche (Paradoxien) verstrickt ist. Solche strukturell bedingten Paradoxien werden als der Kern professioneller Handlungslogik begriffen. Sie begleiten das professionelle Han-

$20 \mathrm{Vgl.} \mathrm{Schütze} \mathrm{(1992).}$

$21 \mathrm{Vgl}$. Dewe/Ferchhoff/Radtke (1992: 14) sowie insbesondere Oevermann (1996).

$22 \mathrm{Vgl}$. insbesondere Stichweh (1992).

23 Organisationen sind immanent darauf angelegt, Verhalten zu programmieren, d.h. Verhalten von seiner Tendenz zu befreien, immer auch anders ablaufen zu können (vgl. grundlegend Luhmann 1969). 
deln nicht bloB, sondern sind dessen konstitutiver Bestandteil und konkretisieren sich (immer wieder und immer wieder in anderer Form) in und durch die Interaktion zwischen Professionellem und Klient. Paradoxien professionellen Handelns konkretisieren sich also durch immer wieder zu treffende, die widersprüchliche Struktur nie endgültig auflösende, praktische Entscheidungen und Verhaltensweisen des Professionellen im Rahmen eines Möglichkeitsraums strukturell bedingter, sich widersprechender Handlungsorientierungen: ${ }^{24}$

- Der Professionelle muss dem Klienten einerseits das Gefühl vermitteln, ihn als, ganze Person' (Diffusität) ,ernst zu nehmen' (d.h. ihn in seiner Individualität wahrzunehmen, sich auf seine, subjektive Geschichte ,einzulassen“, ihn ,verstehen' etc.). Zugleich handelt er jedoch in einer funktionsspezifischen Berufsrolle (Spezifität) , welche ihn ,anhält" nur jene Aspekte der Person ,zuzulassen“, welche der ,Sache“ (Versachlichung und Verunpersönlichung der Beziehung) dienlich sind (etwa: thematische Fokussierung in ärztlichen Anamnesegesprächen).

- Affekte müssen einerseits kontrolliert, andererseits jedoch auch, dosiert' zugelassen werden. Die Aufrechterhaltung affektiver Neutralität gewährleistet die für professionelles Handeln notwendige Sachbindung sowie Problemfokussierung und -objektivierung, während das kontrollierte Zulassen von Affekten aufseiten des Professionellen der Etablierung bzw. Pflege der ebenfalls notwendigen Vertrauensbeziehung zwischen Professionellen und Klient dient.

- Der Professionelle muss sich in die lebensweltliche Situation des Klienten ,hineindenken " (Partikularismus), diese aber zugleich (wissenschafilich) verallgemeinern (Universalismus). Er muss - immer wieder von Neuem (je nach Fall) - allgemeines und abstraktes (theoretisches) Wissen fallspezifisch ,herunterbrechen', d.h. für den jeweiligen, individuellen Fall ,anwendbar machen'. Hiermit einher geht die Schwierigkeit, dass professionelle Deutungen einer theoretischen Einstellung und einer Tendenz zur Verallgemeinerung (etwa: ,Krankheitskarrieren') unterliegen, welche Klienten (,der Praxis') ,vermittelt', d.h. (zumindest teilweise) verständlich (i.S.v. einsichtig) gemacht werden müssen. M.a.W.: Der Professionelle strebt eine Verständnis- und Verständigungsorientierung bei gleichzeitiger methodischer Distanzierung von ursprünglicher Gemeinschaftsbindung an.

- Insbesondere dieser Vermittlungsproblematik entspringt das Schwanken des Professionellen zwischen der Inanspruchnahme zugeschriebener, traditionaler Autorität (ascription) einerseits und der Verpflichtung gegenüber leistungs- und sachgebundener Fachautorität (achievement) andererseits. Prinzipiell ist es die Aufgabe des Professionellen, sein Wissen (an sachfremde Personen/Laien) so zu vermitteln, dass etwaige Lösungen nachvollziehbar werden (idealiter haben sich die ,Vorschläge' des Professionellen über die Logik des besseren Arguments zu legitimieren). Allerdings ist der Professionelle zugleich verpflichtet, dem Klienten (in dessen eigenem Sinne) die ,beste Lösung ', autoritativ ' zu vermitteln, was im Extremfall dazu führen kann, dass Lösungen nicht argumentativ vermittelt, sondern autoritär verordnet werden. Solche Unterminierungen professioneller Handlungslogik können sich verselbständigen und - aufgrund ihres die Praxis vereinfachenden Zugs - habitualisieren (im Falle des ärztlichen Berufsstandes: „Halbgötter in WeiB').

24 Zum Folgenden vgl. insbesondere Brunkhorst (1992). 
- Zudem schwankt der Professionelle zwischen der Orientierung an seinen eigenen Interessen (Geldverdienen, Karriere = Selbstorientierung) auf der einen Seite und der Orientierung am Wohl des Klienten (Kollektivitätsorientierung) auf der anderen Seite.

Die Interaktion zwischen Professionellem und Klient lässt sich vor diesem Hintergrund als die temporäre, zweckgebundene Etablierung einer ,widernatürlichen' Beziehung begreifen, welche im Dienste ihres immanenten Ziels (Wiederherstellung der autonomen Lebenspraxis des Klienten) kurzzeitig so tut (so tun muss), als ob sie eine ,natürliche' sei. Dies hat zur Folge, dass professionelles Handeln, um überhaupt als professionelles gelten zu können, ,Abstand halten' muss zu ,benachbarten' Handlungsformen, in die es aufgrund seiner paradoxalen Anlage immer Gefahr läuft ,umzuschlagen":

- Distanz zum Markt:

- Aufseiten des Professionellen: Notwendigkeit zur ,Verschleierung' der instrumentellen Aspekte der Berufsrolle (Geld verdienen, Karriere) und Simulation einer ganzheitlichen Beziehung (Interesse am Menschen, Vertrauensbeziehung).

- Aufseiten des Klienten: Notwendigkeit eines Eingeständnisses der eigenen Hilfebedürftigkeit und Annahme der Hilfe des Professionellen, was prinzipiell ein ,Sich-Einlassen" auf eine nicht an einer reinen Dienstleitung (als Kunde) orientierten Sozialbeziehung (Vertrauensbeziehung) mit sich bringt.

- Hieraus folgt: Professionelles Handeln lässt sich nicht auf ökonomisches Handeln und die zugrundliegende Beziehungsform nicht auf ein Verhältnis zwischen Dienstleister und Kunde reduzieren.

- Distanz zur Lebenswelt:

- Notwendigkeit (aufseiten des Professionellen) die Tendenz zu regulieren, partikulare Verhältnisse in universelle Solidaritätsbeziehungen hineinzuprojizieren. Professioneller und Klient müssen einen hinreichenden Abstand zur Lebenswelt etablieren, um die ,therapeutische Struktur' (die eben genau darin besteht, dass trotz der strukturellen Asymmetrie eine lebensweltliche Sozialbeziehung simuliert wird) als Voraussetzung für ein Gelingen des ,Projekts' nicht zu zerstören.

- Hieraus folgt: Professionelles Handeln geht nicht im alltäglichen Handeln auf und die zugrundliegende Beziehungsform lässt sich nicht auf ein Freundschafts- oder Verwandtschaftsverhältnis erweitem.

- Distanz zur Bürokratie:

- Aufgrund des Zwangs, wissenschaftliches Wissen unter Handlungsdruck anzuwenden, muss der Professionelle die Tendenz regulieren, Wissen einer Dogmatisierung und Technologisierung zu unterwerfen.

- Hieraus folgt: Professionelles Handeln erschöpft sich nicht in administrativem Verwaltungshandeln und die zugrundeliegende Beziehungsform lässt sich nicht auf ein Verhältnis zwischen Beamtem/Dienstleister und Antragsteller/Leistungsempfänger reduzieren.

Da professionelles Handeln auf diese Weise darüber bestimmt ist, innerhalb paradoxaler Strukturen zu verlaufen, besteht eine ständige (latente) Gefahr, dass die Professionslogik durch Markt, Bürokratie und/oder Lebenswelt zerstört wird.

- ,Gefahren' durch, den Markt':

- Instrumentalisierung (aufseiten des Professionellen): Die Eingebundenheit in die ,kapitalen` Aspekte der Berufsrolle (Gelderwerb, Prestigeerwerb, Karrierestreben) über- 
lagern eine Kollektivorientierung dahingehend, dass das Wohl der Klientel nicht als Hauptanliegen, sondern als Mittel zum Zweck erscheint.

- Ökonomisierung (aufseiten des Klienten): Die Sperrigkeit ,höhersymbolischer` Deutungen führt zu einer (latenten) Ablehnung professioneller Deutungsangebote, so dass es weder zu einem Eingeständnis der eigenen Hilfsbedürftigkeit noch zu einer Akzeptanz der professionellen Hilfe kommt. Vielmehr besteht eine Tendenz, die eigenen Bedürfnisse im Sinne einer geldlich erworbenen Dienstleitung mit fremder Unterstützung zu befriedigen.

- ,Gefahren' durch , die Lebenswelt': Die der Wissensasymmetrie zwischen Professionellem und Klient prinzipiell entspringende Tendenz, dass ,höhersymbolische‘ Deutungen aufseiten von Klienten nicht verstanden, missverstanden oder abgelehnt (Aversion) werden, fördert die Neigung aufseiten des Professionellen, sich der Lebenswelt des Klientel soweit anzunähern ( ,Proletarisierung'; going native), dass ,höhersymbolische` Deutungen (v.a. diskrepanter, den Alltag irritierender Art) nicht vermittelt und statt dessen Deutungsmuster des Alltags in professioneller Atmosphäre ,gedoppelt' (paraphrasiert) werden.

- ,Gefahren' durch, die Bürokratie': Professionelles Handeln erfolgt gemäß festgelegter Wenn-dann-Schemata (Technologisierung) und erschöpft sich damit im Durchführen bewährter Verfahren (Routinenexekution). Die je individuelle Besonderheit des Einzelfalls bleibt unberücksichtigt, (kommt nicht ,zur Sprache') und fällt der (vorschnellen) Einordnung in präformierte Kategorien zum Opfer (Subsumption statt hermeneutische Fallrekonstruktion). Wissenschaftliches Wissen wird nicht eingesetzt, um ,die Praxis` einer ,höhersymbolischen' Deutung zu unterwerfen, vielmehr degeneriert es zur Legitimationsinstanz ,eingefahrener' Berufspraxis oder administrativer Akte.

Hieraus folgt: Hilfsangebote, welche als reine Dienstleistungen, als Routineexekutionen oder als Paraphrasierungen alltäglicher Deutungsmuster auftreten, sind keine professionellen Hilfen in dem hier verstandenen (strukturtheoretischen) Sinn. Hierauf ist sowohl die Anfälligkeit (im berufspraktischen Handeln) als auch die (normative) Sperrigkeit (mit Blick auf (sozial-)politische ,Programme‘) professionalisierter Bereiche für Prozesse der Ökonomisierung/Privatisierung, Bürokratisierung und Veralltäglichung zurückzuführen.

Die Beziehung zwischen Professionellem und Klient, die auf diese Weise etabliert und aufrechterhalten wird (aller Paradoxien, ,Widernatürlichkeiten' und Gefahren zum Trotz), beruht auf kontinuierlichen (Kommunikations-)Leistungen der Akteure (als Beziehungspartner). Sie besteht in wechselseitig kooperativem, an der temporär etablierten ,gemeinsamen Sache` orientiertem Verhalten. M.a.W.: Voraussetzung für das Gelingen jeden professionellen Handelns ist ein ,funktionierendes' Arbeitsbündnis ${ }^{25}$ zwischen zwei konkreten, in Interaktion aufeinander bezogene Personen, dem Professionellen und seinem Klient.

25 Grundlegend für das professionelle Handeln ist - Oevermann (1996) zufolge - die spezifische Strukturlogik der therapeutischen Konstellation, die darin besteht, dass Therapeut und Patient eine Beziehungspraxis zur Wiederherstellung lebenspraktischer Autonomie des Patienten (im Gegensatz zur Zielsetzung der sozialen Kontrolle) konstituieren, die Oevermann (1996) das Arbeitsbündnis nennt. Entscheidend für das Arbeitsbündnis ist, dass zum Erreichen der Zielsetzung (Autonomie) eine widersprichliche Einheit aus Autonomie und Abhängigkeit des Patienten einerseits sowie diffuser und spezifischer Sozialbeziehung andererseits konstituiert und aufrechterhalten werden muss. Der Patient begibt sich also autonom handelnd in eine befristete Abhängigkeit, die gerade darin besteht, seine beschädigte Autonomie wiederherzustellen. 


\section{Pädagogische Professionalität}

Die Formulierung einer genuin pädagogischen Professionalität nimmt einen Sonderweg, welcher sich dadurch auszeichnet, dass man sich vom (unfruchtbaren) Vergleich mit den klassischen Professionen, durch welchen pädagogische Berufe als Semi-Professionen erscheinen, zu emanzipieren versuchte, um einen eigenständigen, der Logik pädagogischen Handelns angemessenen Weg zu finden. Dieser Weg wird jedoch in einer eigentümlichen Doppelbewegung beschritten: Einerseits geht es darum, die signifikanten Differenzen zu den klassischen Professionen (s. Abschn. 2.1) nicht als defizitär, sondern als different und damit als Konstitutivum einer spezifisch pädagogischen Professionalität zu begreifen. Andererseits soll jedoch zugleich plausibilisiert werden, dass es sinnvoll ist, pädagogische Berufstätigkeiten innerhalb der durch handlungs- und strukturtheoretische Ansätze umrissenen Professionslogik (s. Abschn. 1.2) zu verorten (Abschn. 2.2). Als Folge dieser gleichzeitig vollzogenen Abwendung vom und Hinwendung zum Konzept der klassischen Professionen ergibt sich eine Figur, welche als typisch für pädagogische Professionalität gelten kann: das Aufrechterhalten einer (strukturtheoretisch verstandenen) Professionslogik unter erschwerten Bedingungen. ${ }^{26}$

\subsection{Der (unfruchtbare) Vergleich mit den klassischen Professionen}

Misst man pädagogische Berufstätigkeiten am Kriterienkatalog klassischer Professionen, so fällt auf, dass diese einerseits Gemeinsamkeiten (s. Schaubild 2) aufweisen, andererseits jedoch in bedeutsamen Hinsichten vom Idealtypus klassischer Professionen (s. Abschn. 1.1) abweichen. Insbesondere die folgenden Punkte dien(t)en (immer wieder) dazu, pädagogischen Berufen den Status einer (,echten') Profession abzusprechen:

- Fehlende Staatsautonomie:

- Pädagogische Berufe entstehen im Zuge der Industrialisierung als Teil der staatlichen Ordnungsmacht. Sie sind aufgrund dessen von vorneherein mit staatlichen Institutionen verwoben und so nie allein an pädagogischen Standards orientiert.

- Der überwiegende Teil der Berufspädagogen ist organisational in Bürokratien eingebunden (Staat, Verbände) und damit einem ,Dienstherren' gegenüber weisungsgebunden.

- Ihre berufliche Stellung ist demzufolge die eines Angestellten bzw. einer Beamtin und nicht die eines Freiberuflers oder einer Selbstständigen.

- ,Doppeltes Mandat': Der pädagogisch Professionelle handelt infolge dessen sowohl zum Wohle des Klienten als auch im Interesse seines Dienstherren (i.d.R. der Staat).

- Eingeschränkte Klientenautonomie in doppelter Hinsicht:

- Die Abgrenzung gegenüber Laien ist oft unscharf (Ehrenamt).

- Fehlende Freiwilligkeit bzw. Zwangsverpflichtung der Klientel (z.B. Schulpflicht).

- Keine vollausgebildete, eigenständige Fachlichkeit:

$26 \mathrm{Vgl}$. etwa das Konzept der ,bescheidenen Profession` bei Schutze (1992). 
- Fehlen einer eindeutigen Bezugswissenschaft: Bezug auf ,fremde' Fächer (etwa im Falle des Gymnasiallehrers, welcher sich durch den Bezug auf Einzelwissenschaften (etwa die Philologien oder die Geschichtswissenschaft) fachlich legitimiert, nicht aber etwa durch den Bezug auf die Pädagogik).

- Technologiedefizit: In Zusammenhang hiermit wird häufig das sog. „Technologiedefizit" ${ }^{27}$ erwähnt, welches besagt, dass die Leistungen, welche insbesondere durch pädagogische Berufstätigkeiten erbracht werden, nämlich Personenveränderungen, an Kommunikationen mit diesen Personen gebunden sind und deshalb nicht (i.S.e. Technologie) kausalgesetzlich verfasst sein können (s.a. Glossar zu den Stichworten „Normativitätsproblem“" sowie „Zielkompetenz").

- Unscharfer Fallbezug:

- Prospektive Handlungslogik: In den meisten Fällen ist pädagogisches Handeln an der (erstmaligen) Erzeugung von Norm- und Wertorientierungen bzw. an der Abwendung verhinderungswürdiger Zustände und Entwicklungen (Präventionslogik) ausgerichtet. Im Gegensatz zu einer retrospektiven Handlungslogik, welche an der Wiederherstellung lebenspraktischer Handlungsautonomie orientiert ist.

- Kein ,direkter' Leidensdruck: Demzufolge fehlt innerhalb pädagogisch-professionellen Handelns häufig eine dem Leidensdruck analoge Motivation, eine temporäre Abhängigkeit im Rahmen einer (Vertrauens-)Beziehung zu einem ,Experten` einzugehen. M.a.W.: Pädagogisierte empfinden sich i.d.R. selbst nicht als (professioneller) Hilfe bedürftig.

- Kein dyadischer Fallbezug: Zudem bezieht sich pädagogisches Handeln in der Berufpraxis häufig auf Gruppen, so dass eine Zweierbeziehung (Dyade) als ideale Form der Fallbearbeitung (Prototyp: Therapie) zwischen Professionellem und Klient nicht zustande kommt.

- Doppelter Klientenbezug: Häufig haben es pädagogisch Handelnde darüber hinaus nicht bloß mit einem mündigen Klienten zu tun, sondern i.d.R. mit (mehr oder weniger) unmündigen Klienten (alters- und/oder krankheitsbedingt), welche ihrerseits durch Sorgeberechtigte (Eltern, Vormünder) vertreten werden.

Pädagogische Berufstätigkeiten - so legt dieser Vergleich nahe - weisen hinsichtlich ihres Professionalisierungsgrades Defizite auf. Aufgrund solcher ,Mängel erschienen pädagogische Berufe als nur teilweise oder ,halb'-professionalisierte Tätigkeiten, was im Konzept der ,Semi-Professionen' seinen Ausdruck fand. ${ }^{28}$

27 Vgl. Luhmann/Schorr (1982: 11 ff.). Ein Technologiedefizit wäre allerdings bereits im Falle der klassischen Professionen zu veranschlagen. Auch der Arzt - so zeigt Parsons (1965) - handelt prinzipiell auf unsicherer Informationsbasis und muss in der Praxis riskante Entscheidungen treffen. Von einer ,Technologie' i.e.S. kann also auch im Falle der klassischen Professionen keine Rede sein, weshalb es sich hier eher um eine graduelle Unterscheidung handelt, welche sich auf die Differenzierung ,harter' (Naturwissenschaften) und ,weicher ' (Sozial- und Geisteswissenschaften) Wissenstraditionen bzw. Wissenschaftsdisziplinen stützt.

$28 \mathrm{Vgl}$. Dewe/Otto (2001: 1405). 


\section{Eigenständigkeit pädagogischer Professionalität}

Das Konzept der Semi-Profession erwies sich als Sackgasse für die Entwicklung eines genuin pädagogischen Professionalisierungskonzeptes. ${ }^{29}$ Nicht die (unmögliche) Überwindung des indikatorisch festgestellten ,Abstands' zu den klassischen Professionen, sondern die Fokussierung der inneren Logik pädagogischen Handelns unter den ihr ganz eigenen Strukturbedingungen sollte den Schlüssel zu einer eigenständigen Bestimmung pädagogischer Professionalität darstellen. Ausgangspunkt ist (nach wie vor) die Bezugnahme auf den Diskurs um eine De-Pädagogisierung der Erziehung durch Verberuflichungsprozesse ${ }^{30}$, welcher - grob gesprochen - eine Fragmentierung des ursprünglich ganzheitlichen pädagogischen Bezugs beklagt. Innerhalb strukturtheoretischer Professionalisierungsansätze geschieht dies allerdings mit dem Ziel, Extrempositionen bzw. Verkürzungen zu vermeiden, d.h. eine solche Diagnose nicht zum Anlass zu nehmen, Verberuflichungsprozesse aufgrund ihrer ,de-pädagogisierenden ' Wirkung ,zurückdrehen ' $z u$ wollen ${ }^{31}$ bzw. aufgrund der verloren gegangenen Ganzheitlichkeit Erziehungsansprüche zu relativieren ${ }^{32}$, sondern als Ausgangspunkt für eine Bestimmung pädagogischer Professionalität zu nehmen. Unhintergehbarer Ausgangspunkt einer pädagogischen Professionalität ist dann a) ihre Berufsförmigkeit (als notwendiges Moment der Abgrenzung von Primärerziehung und Laienschaft) sowie b) der Erziehungsanspruch (als regulative Leitidee) pädagogischen Handelns. Als Folge sind (notwendige) Widersprüchlichkeiten zu veranschlagen, welche im Spannungsfeld von Ganzheitlichkeitsansprüchen und Fragmentierungen (in Hinblick auf die pädagogische Beziehung) bzw. von erzieherischen Ansprüchen auf Normvermittlung und Prozessen der Wertepluralisierung (in Hinblick auf Erziehungsziele), aufbrechen'.

Eine weitere Verkürzung betrifft die Vorstellung, wissenschaftlich erworbenes Wissen sei dergestalt ausnutzbar, dass praktische Verfahren und Prozeduren deduzierbar wären, welche durch ,richtige“ Anwendung kalkulierbare Resultate erzeugten. Die Vorstellung einer, Sozialtechnologie - verdichtet im Idealtypus des Sozialexperten - erwies sich ebenfalls als irrig. ${ }^{33}$ Hiermit verbunden ist insbesondere die Akzeptanz des sog. Technologiedefizit (s.o.) als konstitutiver Bestandteil (und nicht etwa als Defizit) pädagogisch-professionellen Handelns: Im Gegensatz zu expertokratischem Handeln in instrumentellen Kontexten, wo Erklärungswissen in eindeutiger Weise (gelingende) Anwendungen erzeugt (etwa: beim Ingenieur oder in der ,Apparate-Medizin'), hat es pädagogisches Handeln nicht mit sinnfremden Gegenständen (Maschinen, Körpern), sondern mit Menschen in kommunikativen Kontexten zu tun, welche auf ,Anwendungen' unterschiedlich reagieren. Aufgrund dessen können die Folgen der Anwendung theoretischen Wissens nicht im Vorfeld ,berechnet' und festgelegt werden, d.h.: Der Prozess der Wissensanwendung ist nicht ,technologisierbar'. Gefragt ist damit interpretatives Sinn- und Bedeutungsverstehen, also der jeweils situative, fallabhängige bzw. -begleitende und deshalb sich immer wieder anders gestaltende Rekurs auf theoretisches Wissen. ${ }^{34}$

$29 \mathrm{Vgl}$.zum Folgenden insbesondere Dewe/Ferchhoff/Radtke (1992)

$30 \mathrm{Vgl}$. Dewe/Ferchhoff/Radtke (1992: $8 \mathrm{ff}$.).

$31 \mathrm{Vgl}$. etwa Fischer (1950).

32 Vgl. etwa Giesecke (1996).

33 Vgl. hierzu Dewe/Ferchhoff/Radtke (1992: $26 \mathrm{ff}$.).

$34 \mathrm{Vgl}$. Dewe/Ferchhoff/Radtke (1992: 9 f. sowie insbesondere $26 \mathrm{ff}$.) sowie Ferchhoff (1993). 
Die folgende Aufzählung verweist auf gängige Versuche, die dem berufsförmig pädagogischen Handeln inhärenten Spannungen aufzulösen, worin sich unschwer die oben aufgezeigten, idealtypischen ,Gefahren' einer Professionslogik (Abschn. 1.2) wieder erkennen lassen $^{35}$ :

- Pädagogik als Berufung (Rückkehr zum ganzheitlichen pädagogischen Bezug; Rekurs auf intuitives, wissenschaftsfernes Wissen; prototypische Figuren: der ,geborene Erzieher', der ,professionelle Altruist').

- Gefährdung durch zu große Nähe zur Lebenswelt (,Proletarisierung‘; s.o.)

- Pädagogik als Sozialtechnologie (expertokratische Wissensanwendung; prototypische Figur: der ,Sozialingenieur')

- Gefahr durch Technologisierung und Bürokratisierung

- Pädagogik als Dienstleistung (Klientel wird zur Kundschaft und bestimmt selbst, welche Angebote es erwerben möchte; der Professionelle wird zum bezahlten ,Erfüllungsgehilfen')

- Gefahr durch zu große Nähe zum Markt.

Unter handlungslogischer bzw. strukturtheoretischer Perspektive erscheinen pädagogische Berufstätigkeiten dagegen prinzipiell als professionalisierbar bzw. - empirisch betrachtet u.U. als professionalisierungsbedürftig, was auch bedeutet, ihnen ist eine handlungslogische Homologie (bei allen sonstigen Verschiedenheiten; s. Abschn. 2.1 sowie Schaubild 2) mit den klassischen Professionen zu unterstellen. D.h., dass

nicht mehr nur die sozialen und ökonomischen Dimensionen der Verberuflichung, sondern die Strukturprobleme pädagogischen Handelns ins Zentrum der Aufmerksamkeit rückt. Sie wird nicht mehr vornehmlich als legitimations- und standespolitische Debatte im Sinne eines Aufstiegsprojekts geführt oder in der Perspektive einer Effektivierung und Rationalisierung von pädagogischen Prozessen, sondern sie nähert sich tastend den Binnenstrukturen und der Logik pädagogischen Handelns im Spannungsfeld von allgemeiner Wissensapplikation und situativem Fallverstehen. (Dewe/Ferchhoff/Stüwe 1992: 194)

Als allgemeinste Ableitungsbasis pädagogischer Professionalität können daher strukturtheoretische Konzepte (s. Abschn. 1.2) veranschlagt werden. Demzufolge lässt sich pädagogische Professionalität zunächst - ganz ähnlich der Bestimmung klassisch professionellen Handelns - über das Konzept der stellvertretenden Deutung bestimmen, welche sich - wie oben erörtert - im Spannungsfeld „von universalisierter Regelanwendung und striktem Fallbezug“ (Dewe/Ferchhoff/Radtke 1992: 14) bewegt. Ferchhoff (1993) bringt dies auf die prototypische Figur des „,stellvertretenden Lebenslagen- und Lebenswelthermeneuten“.

Ist pädagogische Professionalität auf diese Weise als prinzipiell professionalisierbare bzw. -bedürftige Berufstätigkeit bestimmt, geht es im nächsten Schritt darum, Gemeinsamkeiten und Differenzen zur Primärerziehung auf der einen und den klassischen Professionen auf der anderen Seite in systematischer Weise herauszustellen, um sie als Grundlage und Ausgangspunkt einer Strukturlogik pädagogischer Professionalität zu nehmen. Dies soll im Folgenden, mithilfe einer tabellarischen, die bisherigen Erkenntnisse zusammenfassenden Übersicht geschehen: ${ }^{36}$

35 Zum Folgenden vgl. insbesondere Ferchhoff (1993).

$36 \mathrm{Vgl}$. zum Folgenden insbesondere Dewe/Ferfchhoff/Radtke (1992: 12 ff.). 


\begin{tabular}{|c|c|c|}
\hline & Gemeinsamkeiten & Differenzen \\
\hline 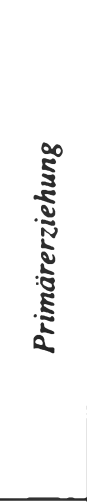 & $\begin{array}{l}\text { - Grundstruktur: } \\
\text { „(Als bereits sozialisiert } \\
\text { geltende) Erwachsene } \\
\text { suchen noch nicht Sozi- } \\
\text { alisierte oder aufgrund } \\
\text { angebbarer Ereignisse, in } \\
\text { ihrer Handlungsautonomie } \\
\text { Beeinträchtigte‘ in Rich- } \\
\text { tung auf bestimmte Ziele zu } \\
\text { erziehen“ (ebenda: 15). }\end{array}$ & $\begin{array}{l}\text { - Wissenschaftlich generiertes Sonderwissen } \\
\text { - Reflexiver Wissens- und Handlungstypus (Wissen über die } \\
\text { zu steuernden Prozesse zur (nachträglichen) Evaluation und } \\
\text { Kontrolle dieser Prozesse) } \\
\text { - Organisational verfasst (institutionelle Rahmung symboli- } \\
\text { siert den Anspruch auf außeralltägliche Problembewältigung, } \\
\text { findet in spezifischen Räumlichkeiten statt und folgt speziellen } \\
\text { Regeln; eingespielte Praktiken sind auf diese Weise konventi- } \\
\text { onell abgesichert) } \\
\text { - Hiermit geht einher: Selbstdeklarierung der Situation als } \\
\text { einer pädagogischen, die von den zu Erziehenden anerkannt } \\
\text { werden muss }\end{array}$ \\
\hline 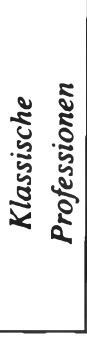 & $\begin{array}{l}\text { - Zentralwertbezug: } \\
\text { Erziehung als Grund- } \\
\text { konstante menschlicher } \\
\text { Gesellschaften } \\
\text { - Vergleichbare Handlungs- } \\
\text { logik: Stellvertretende Deu- } \\
\text { tung bzw. verwissenschaft- } \\
\text { lichte Krisenbewältigung }\end{array}$ & $\begin{array}{l}\text { - Fehlende Staatsautonomie („,doppeltes Mandat“) } \\
\text { - Eingeschränkte Klientenautonomie } \\
\text { - Eingeschränkte fachliche Eigenständigkeit } \\
\text { - Unscharfer Fallbezug (prospektive Handlungslogik, Fehlen } \\
\text { eines ,direkten` Leidensdrucks, kein dyadischer Fallbezug, } \\
\text { doppelter Klientenbezug) } \\
\text { - Folgen: gesteigertes Technologiedefizit, gesteigertes Norma- } \\
\text { tivitätsproblem, gesteigerte Begründungsverpflichtung }\end{array}$ \\
\hline
\end{tabular}

Schaubild 2: Eckpunkte der Bestimmung professionell-pädagogischen Handelns - Gemeinsamkeiten und Differenzen zur Primärerziehung und den klassischen Professionen

Aus dieser besonderen Konstellation pädagogischer Berufstätigkeiten ergeben sich Paradoxien, welche professionell-pädagogisches Handelns im Allgemeinen (also nicht handlungsfeldspezifisch, s. hierzu Abschn. 2.3) begleiten. Teilweise erscheinen sie als Radikalisierungen klassisch-professioneller Paradoxien (s. Abschn. 2.1). Insbesondere der Umstand, dass pädagogisches Handeln sowohl institutionellen Interessen als auch Bedürfnissen der Klientel folgt (,doppeltes Mandat'), erzeugt ein gesteigertes Normativitätsproblem, welches darin besteht, dass Maßnahmen schwieriger zu vermitteln sind und damit in aufwändigerer Weise begründet und abgesichert werden müssen. Gründe hierfür sind zum ersten die z.T. unscharfe Abgrenzung gegenüber Laientätigkeiten sowie - v.a. in der Sozialarbeit/Sozialpädagogik - eine diffuse Allzuständigkeit im Verbund mit kaum greifbaren (,messbaren') Handlungserfolgen und Problemlösungen.$^{37}$ Erschwerend kommt zweitens der uneindeutigere Fallbezug, insbesondere der fehlende Leidensdruck hinzu, was dazu führt, dass Klienten professionell-pädagogische Hilfe (häufig) nicht freiwillig aufsuchen, sondern sich die Hilfe in (mehr oder weniger) zwangsverpflichteten Formen bewegt ${ }^{38}$,

37 Dies gilt im Besonderen für nur sehr diffus institutionalisierte Bereiche der Sozialpädagogik, wie etwa die Jugendarbeit (vgl. Münchmeier 1980).

38 Dewe/Ferchhoff/Radtke (1992) sprechen etwa davon, dass „Gruppen, die zum Zwecke der Erziehung formiert werden, (...) rechtlich (...) oder virtuell gezwungen [sind], das Angebot wahrzunehmen“ (15). 
wodurch neben eine wissens- auch eine machtbasierte Asymmetrie tritt. Der strukturelle Mangel an Freiwilligkeit ist Quelle für Widerständigkeiten (aufseiten des Klientel) und Ritualisierungen (pädagogisch-institutionelle Interaktionen betreffend), was wiederum Begründungsbedarf (im Sinne einer demokratischen, kooperativen Vermittlung von Deutungsangeboten als regulativer Leitidee) erzeugt, um Verständigungssicherung herzustellen. Ist Verständigung (und damit Kooperation und Akzeptanz) nicht zu erreichen, besteht die Gefahr, dass pädagogisches Handeln in rein ,autoritative', normsichernde Handlungsformen ,umschlägt' oder, gesteckte' Ziele aufgibt (was beides de-professionalisierende Wirkung hätte) bzw. modifiziert, also den Bedürfnissen des Klientel soweit anpasst, dass eine Verständigungssicherung zu erreichen ist (was jedoch letztlich wiederum an (institutionelle) Grenzen stößt und den dann erhöhten Zwang erzeugt, Zielerreichung unter Rückgriff auf organisational verbürgte Macht sicherzustellen). Kurz: Gesteigerte Normativitätsproblematik sowie - damit zusammenhängend - gesteigerte Begründungsverpflichtung aufgrund eingeschränkter (Handlungs-)Autonomie und Fachautorität sowie mangelndem Fallbezug verstärken das oben erwähnte Technologiedefizit dahin gehend, dass pädagogische Maßnahmen in besonderer Weise einer Vermittlungsproblematik unterworfen sind. Paradoxien pädagogischen Handelns können vor diesem Hintergrund auf folgende Kernproblematiken verdichtet werden:

- Pädagogisch-professionelles Handeln bewegt sich im Spannungsfeld von Verständigungssicherung, Druck- und Zwangsausübung sowie an den Bedürfnissen des Klientel orientierten (Erziehungs-)Zielmodifikationen.

- Pädagogisches Handeln hat es daher besonders schwer, sich gegenüber seiner Klientel zu legitimieren resp. eine auf freiwillige Mitarbeit gründende Vertrauensbeziehung aufzubauen und

- schwankt deshalb immer (mehr oder weniger) zwischen administrativ(-rechtspflegerischem), an staatlichen Vorgaben orientiertem Handeln (soziale Kontrolle) auf der einen und personen-bzw. klientenorientiertem, hermeneutisch-fallspezifischem Handeln (Klientenwohl/Bedürfnisorientierung) auf der anderen Seite.

- Aufgrund dessen besteht eine beständige (strukturell bedingte) Zieldiskrepanz zwischen Wiederherstellung der Autonomie der Lebenspraxis (Fokus: Wohl des Individuums) und normensicherndem Rechtshandeln (Fokus: Gesellschaftsintegration).

\subsection{Handlungsfeldspezifische Aufgaben, Paradoxien und kommunikative Bewältigungsstrategien}

Neben dieser allgemeinen und abstrakten, strukturtheoretischen Bestimmung pädagogischer Professionalität, welche darauf zielt „durch die je besonderen Erscheinungen hindurch die Einheit aller erzieherischen Praxen, das Proprium der Erziehung, aufzuspüren (Dewe/Ferchhoff/Radtke 1992: 16), lässt sich „das Besondere des pädagogisch-professionellen Handlungsmodus “ (ebenda) darüber rekonstruieren, dass spezifische Aufgabenstellungen sowie sich daraus ergebende Paradoxien und Bewältigungsformen unterschiedlicher pädagogischer Berufstätigkeiten entlang der Ausdifferenzierung pädagogischer Felder aufgezeigt werden. 
Die Forderung strukturtheoretisch informierter Konzepte pädagogischer Professionalität, die Logik pädagogischen Handelns anhand von Rekonstruktionen pädagogischer Interaktionen, d.h. anhand konkreter Fälle aus der Praxis, zu bestimmen, ist bereits vielfach umgesetzt worden ${ }^{39}$ Empirische Analysen handlungsfeldspezifischer Aufgabenstellungen, Paradoxien und Bewältigungsformen liegen für einzelne pädagogische Handlungsfelder in umfänglicher Form vor (insbesondere für die Felder der ,Schule' und ,Sozialarbeit/Sozialpädagogik'), für andere dagegen weit weniger (etwa ,Offene Jugendarbeit'). Im Folgenden sollen zentrale Resultate einschlägiger Analysen vorgestellt werden. Der Schwerpunkt soll dabei auf das Lehrerhandeln im Unterricht gelegt werden (Abschn. 2.3.1). Abschließend soll zum Vergleich kurz auf typische Handlungsanforderungen und -widersprüchlichkeiten in der Sozialarbeit/Sozialpädagogik (Abschn. 2.3.2.) eingegangen werden, um deutlich zu machen, dass und inwiefern professionelle Handlungslogiken bereichsspezifisch variieren.

\subsubsection{Schule}

Das Schulsystem als der ausdifferenzierteste, uniformste und formalisierteste Teil des Erziehungssystems hat die primäre Aufgabe Wissen an zu Sozialisierende (Unmündige, Heranwachsende) zu vermitteln. Schule ist die zentrale Institution der Weitergabe des gesellschaftlich relevanten Wissensvorrates. Dies geschieht im Rahmen bestimmter Interaktions- und Normverhältnisse sowie unter bestimmten Wertprämissen, weshalb Schule auch Sozialisations- und Erziehungsfunktionen erfüllt. ${ }^{40}$ Zugleich stellt das Schulsystem auf der Grundlage der regelmäßigen Durchführung von Lernerfolgskontrollen (etwa Prüfungen) und deren schriftlicher Dokumentation in Form bewertender Kenngrößen (Klausurergebnisse, Zensuren, Zeugnisse, Zertifikate) die Möglichkeit sicher, Schüler leistungsbezogenen zu differenzieren (Selektionsfunktion). Dies ist die Voraussetzung für eine leistungsrelative Verteilung von Personen auf entsprechende Berufspositionen (Allokation). Da es sich hierbei um biographierelevante Größen handelt und es damit um die Verteilung von Zukunftschancen geht, fungieren Zertifizierungen als ,Motivatoren' (Selektionsverstärker) und tragen aufgrund dessen zur Verhaltensregulierung bei. Die Bewältigung dieser Aufgaben erfolgt durch die Institutionalisierung eines Lehrer-Schüler(gruppen)-Interaktionssystems, welches einerseits professionslogische Züge trägt ${ }^{41}$, andererseits jedoch im rechtlichen Rahmen einer Zwangsverpflichtung der Zu-Unterrichtenden statt findet, und damit gegen das für pro-

$39 \mathrm{Vgl}$. etwa Becker-Mrotzek/Vogt (2001), Combe/Helsper/Stelmaszyk (1999), Harrach/Loer/Schmidtke (2002), Krummheuer/Naujok (1999), Nolda (2000), Ohlhaver/Wernet (1999a).

40 So lemen Kinder mit dem Eintritt in die Institution Schule etwa (auch), sich in spezifisch ausgerichteten und an Leistung orientierten Rollenbeziehungen zu bewegen.

41 Dass die Lehrer-Schuler-Interaktionsbeziehung ,professionslogische Züge' trägt, bedeutet, dass sie zunächst und in prinzipieller Hinsicht mit der Arzt-Patient-Beziehung vergleichbar ist (vgl. Brunkhorst (1992: 53 f.). Erst auf der Grundlage einer solchen höherstufigen, solidarischen Verbundenheit - so betont Brunkhorst (1992: 53) - zwischen Lehrpersonal und Schulerschaft ist das Schulsystem in der Lage sowohl Selektions- als auch Sozialisationsfunktionen zu erfüllen. Mehr noch: Es ist die Voraussetzung dafür, das nicht nur inhalttlich vermitteltes Wissen kognitiv angeeignet, sondern auch ,schulische Prinzipien' (etwa: dass Selektionen leistungsbasiert zu erfolgen haben) als Wertmuster verinnerlicht werden. Letzteres - also die Vermittlung gesamtgesellschaftlicher Werte - ist eben nur im Rahmen höherstufiger, universeller Solidarität möglich. 
fessionelles Handeln konstitutive Reziprozitätsprinzip verstößt. Dieser (temporäre) Entzug lebenspraktischer Autonomie wird Brunkhorst (1992: $66 \mathrm{f}$.) zufolge durch die informelle Organisation der Schulklasse (Freundschaften, Cliquen, Gruppensolidarität) entschärft, da diese eine Art ,sekundärer Reziprozität“ (ebenda) hervorbringt. Die Peer-Group (Gruppe der Gleichaltrigen und Gleichgestellten) sichert ihren Mitgliedern auf diese Weise einen Autonomiespielraum gegenüber Ansprüchen der Erwachsenenwelt, insbesondere gegenüber schulischen Forderungen vertreten durch die Lehrerrolle. M.a.W.: Die (relative) Autonomie der Peer-Group sowie der ihr (eingeräumte) Abweichungsspielraum sind Voraussetzung für ein ,funktionierendes“ Arbeitsbündnis zwischen Lehrer und Schülern. Der Lehrer ist aufgrund dieser kompensatorischen Funktion des Gleichaltrigenkollektivs von dessen ,Zustimmung abhängig, er benötigt (ein Mindestmaß an) Kooperation, um Unterrichten und damit seiner Hauptaufgabe nachzukommen zu können. Allerdings bedingt genau diese Konstellation umgekehrt jene schultypischen ,Spaltungen' (Lehrer gegen Schülerschaft) und damit verbundenen Abweichungspotenziale (etwa: ,Störungen des Unterrichts'), welche unter Einsatz ,richtiger' Maßnahmen bzw. kompetent ausgebildeter Personen für eliminierbar gehalten werden. Tatsächlich sind sie im Rahmen schulischer Kommunikation nicht nur unvermeidlich, sondern - wie die obige Erörterung zeigen sollte - vielmehr konstitutiv für deren Gelingen. Da lehrende Tätigkeiten personenbezogen (,am Menschen') erfolgen, also letztlich größtenteils Beziehungsarbeit bedeuten und daher in besonderer Weise auf Kommunikation angewiesen sind, besteht eine Kernproblematik des Schulsystems darin, Kommunikationsprozesse so zu formalisieren, dass größeren Gruppen ein Wissen vermittelt werden kann, dessen Inhalte sie nicht (mit-)bestimmen, dessen Relevanz sie zum gegebenen Zeitpunkt u.U. nicht abschätzen können und dessen sie u.U. eigener Einschätzung nach nicht bedürfen. ${ }^{42}$ M.a.W.: Eine Kommunikationsform, die sicherstellt, dass trotz thematischer Fremdbestimmung (und u.U. mangelnder Einsicht in die Relevanz des Wissens und mangelnder Neugier) die Grundvoraussetzung für eine Vermittlung von Wissen, nämlich das Aufrechterhalten eines thematischen Aufmerksamkeitsfokus, gewahrt ist und bleibt. Dies geschieht durch die Institutionalisierung einer spezifischen Kommunikationsform, der Unterrichtskommunikation, welche die Offenheit (Diffusität) und Beliebigkeit (Kontingenz) direkter, informeller Kommunikation reduziert. Eine solche Formalisierung der Kommunikation wird zwar zum einen durch das besondere Interaktionssystem (s.o.) legitimiert und strukturell abgesichert (v.a. durch fachliche und organisationale Asymmetrie und Autorität), muss jedoch zum anderen kontinuierlich interaktional aufrechterhalten (prozessiert) werden, was durch spezifische Kommunikationsmuster und -regeln sowie deren (metakommunikative) Sicherung geschieht. Hervorzuheben sind hier:

- Gesprächsorganisation: Regelungen des Sprecherwechsels (Wer darf wann reden? Wie wird der nächste Sprecher bestimmt?) sichern zum einen die kommunikative Vormacht-

42 In beiden Fällen lässt sich strukturell eine Analogie zur Professionslogik erkennen: Im Falle des (Des-)Interesses am vermittelten Wissen nimmt die Neugier die systematische Stelle des Leidensdrucks ein (vgl. Oevermann 1996: 152 ff.), d.h. dass ein kooperativitätssichemdes Arbeitsbündnis umso eher zustande kommt, desto ,freiwilliger" sich Lernende in die Situation des Wissenserwerbs begeben. Im Falle mangelnder Einsicht in die Relevanz des vermittelten Wissens, lăsst sich unschwer eine Parallele zur Vorstellung des Mandats, zum Wohle des Klienten zu handeln, erkennen, was aufseiten des Klientel einen Vertrauensvorschuss aufgrund mangelnden Wissens erfordert. 
stellung des Lehrers und garantieren zum anderen - zumindest auf einer formalen Ebene -, dass jeder Redebeitrag von jedem gehört wird. Gelöst wird dieses Problem i.d.R. durch Melde- und Aufrufsysteme, welche nach dem Muster ,Aufruf durch die Lehrperson - Redebeitrag (eines Schülers) - Kommentar/Neuaufruf (durch Lehrperson)` ablaufen. Entscheidend ist, dass ,das Wort' (das Rederecht) nach den Schülerbeiträgen (dies gilt auch im Falle von längeren ,Aufruf-Stafetten` der Schüler untereinander) an die Lehrperson zurückfällt.

- Thematisch, handlungsbezogen: Fokus der Aufmerksamkeit ist ,der Unterricht ', d.h. dass (Sprech-)Handlungsaktivitäten erkennbar am Ziel der Unterrichtskommunikation ausgerichtet sein sollten. Hierzu müssen Gesprächsbeiträge themenbezogen sein und alle ,sonstigen“ Aktivitäten müssen reduziert werden. Alles was ,neben' oder thematisch nicht auf die Unterrichtskommunikation bezogen geschieht, erscheint als ,Störung (s.u.).

- Funktionsbezug: Die Umsetzung der primären Aufgabe von Schule, nämlich die Vermittlung von Wissen durch Wissende an Unwissende (Wissenstransfer auf der Grundlage von Wissensasymmetrien) erfolgt entweder monologisch durch Lehrervortrag (Frontalunterricht) oder dialogisch durch Lehr-Lern-Gespräche.

- Kommunikationsmuster: Letzteres gilt als eigentlicher Kern schulischer, wissensvermittelnder Interaktion, da erst hier ein echter Austausch zwischen Lehrendem und Lernendem (im Sinne eines Wissenstransfers) statt finden $\mathbf{k a n n}^{43}$, welcher sich auf ein dreizügiges Kommunikationsmuster verdichten lässt: ,Frage - Erwiderung - Bewertung ${ }^{\text {(44 }}$.

- Struktursicherungsoperationen: ${ }^{45}$ Hierunter sind Kommunikationsakte zu verstehen, welche die Einhaltung der unterrichtskonstituierenden Interaktionsregeln (etwa des Sprecherwechsels, der Aufrechterhaltung eines Aufmerksamkeitsfokus, des themengebundenen Sprechens) sicher stellen sollen. Ihre Aufgabe ist die Bewahrung funktional relevanter Strukturmerkmale schulischer Kommunikation.

Insbesondere (oben erwähnte) Abweichungspotenziale werden auf der Ebene der Interaktion virulent und können auch vornehmlich nur dort ,behandelt' werden. Sie erscheinen dort als ,Störungen' des Unterrichts, d.h. als (Sprech-)Handlungen, die die funktional auf das Ziel der Wissensvermittlung bezogene Form der Unterrichtskommunikation konterkarieren (etwa unaufgefordertes Sprechen, Nebengespräche etc.). Unterrichtskommunikation wird auf diese Weise störanfällig bzw. sicherungsbedürftig, da Abweichungen i.d.R. nicht einfach ignoriert werden können oder ,nebenher laufen' können, sondern zur Aufrechterhaltung schulischer Unterrichtskommunikation minimiert werden müssen. Kurz: Bevor der eigentliche Unterricht fortgesetzt werden kann, muss die Störung behandelt und beseitigt werden (etwa durch Ermahnungen u.ä.). Typisches Problem schulischer Unterrichtskommunikation ist die Ausbeutung dieser Störanfälligkeit, die dadurch zustande kommt, dass zum einen Autonomiespielräume gewährt werden müssen (s.o.), welche dann zum anderen dergestalt ausnutzbar

43 Sowohl beim Frontalunterricht als auch u.U. bei Gruppenarbeiten fehlen jeweils die Rückmeldungen. Erfolgen Gruppenarbeiten unter bestimmten Aufgabenstellungen und erhalten die Resultate ein Feedback, folgen auch sie dem Muster ,Frage - Erwiderung - Bewertung'. Einen Sonderstatus nehmen ,freie Diskussionen' ein (vgl. Schneider 1997). Unschwer einzusehen ist, dass die schulische Wissensvermittlung aufgrund ihrer notwendigerweise asymmetrischen Anlage nie vollständig und auch nicht überwiegend als ,freie Diskussion` organisierbar ist.

$44 \mathrm{Zu}$ Beispielen s. Becker-Mrotzek/Vogt (2001); Krummheuer/Naujok (1999).

$45 \mathrm{Vgl}$. Schneider (1997). 
werden, dass die Unterrichtskommunikation Gefahr läuft, durch Struktursicherungsoperationen blockiert zu werden, da Störungsbeseitigungen - im Sinne einer Sicherstellung der Grundvoraussetzungen von Unterrichtskommunikation - zeitlich prioritär sind. Schüler wissen, indem sie Störung an Störung reihen, bringen sie das normale Unterrichtsgeschehen zum Erliegen und konterkarieren damit den Sinn von Schule und Unterricht bzw. hindern den Lehrer an der Erfüllung seiner eigentlichen Aufgabe. Da diese Struktur jedoch nicht verhandelbar ist ${ }^{46}, \mathrm{~d} . \mathrm{h}$. Schüler und Lehrer sich im Rahmen schulischen Unterrichts nie in einer symmetrischen Kommunikation begegnen, entsteht die Schwierigkeit, die Kooperation des Gleichaltrigenkollektivs (Schüler) trotz strukturell bedingter Asymmetrien zu erreichen. Der Professionslogik folgend (,zum Wohle des Klienten'), soll dies zudem ohne Zwang, d.h. auf der Basis von Freiwilligkeit und Einsicht geschehen. Das Kernproblem bzw. Paradox schulischer Kommunikation besteht letztlich in der Bewältigung der strukturellen Diskrepanz, Kooperation zu erzielen, ohne echte Mitbestimmung anbieten zu können. ${ }^{47}$ Dieses strukturelle Dilemma, welches letztlich auf den funktional notwendigen Mangel an Reziprozität der Lehrer-Schüler-Beziehung zurückzuführen ist, schlägt sich in der Interaktion (Unterrichtskommunikation) als Abweichung nieder, welche durch den Lehrer (als Bewahrer zentraler institutioneller Funktionen) sowohl zugelassen als auch beschränkt, also ausgehalten und balanciert werden muss. Lehrerprofessionalität - so ließe sich schließen - besteht zunächst v.a. in der kontinuierlichen Bewältigung dieses Strukturkonflikts in und durch Kommunikation.

\subsubsection{Sozialarbeit/Sozialpädagogik}

Im Gegensatz zu Schule ist Sozialarbeit/Sozialpädagogik (im folgenden: SA/SP) funktional nicht darauf angelegt, über die Bewertung von an Wissensvermittlungsprozesse gebundener Leistungen sozialstrukturell und biographisch relevante Differenzen zu produzieren (Selektion/Allokation). Vielmehr folgt die SA/SP der regulativen Leitidee, Gerechtigkeit und Gleichheit herzustellen und zu erhalten bzw. Ungerechtigkeiten und Ungleichheiten zu kompensieren. Der funktionale Fokus der SA/SP liegt damit zum einen auf der Verhinderung von (ungerechten) Ungleichheitszuständen (durch Prävention) sowie zum anderen auf der Wiederherstellung von (gerechten) Gleichheitszuständen (durch Intervention). ${ }^{48}$ Dies

46 Parodien der Sicherungsstruktur schulischer Unterrichtskommunikation (vgl. Schneider 1997) zeigen, dass Schüler nicht nur wissen, wie Störungen aussehen, wie ubblicherweise auf Störungen reagiert wird und welche Konsequenzen solche Störungen langfristig haben, d.h. sich nicht nur mit Form, Funktion und Ablauf dieses Kommunikationstyps sehr gut auskennen, sondern daruber hinaus zu wissen scheinen, dass diese Struktur - als funktional zwingendes Kernelement schulischer Unterrichtskommunikation - rational bzw. argumentativ nicht veränderbar, wohl aber parodierbar und sabotierbar ist. M.a.W.: ,Veränderungen` sind nie strukturell durch Argumentation, sondern immer nur punktuell durch Regression zu erreichen

47 Das institutionalisierte Mitbestimmungseinrichtungen häufig oktroyiert erscheinen und damit jene Struktur, die sie abmildern sollen, selbst wiederum reproduzieren, zeigt etwa Helsper (1996). Selbst- und Mitbestimmungsformen erscheinen dann als „verordnete Autonomie“" (ebenda: 551).

48 Nicht verhinderungswürdig bzw. kompensationsbedürftig sind demzufolge gerechte Ungleichheitszustände, wie sie etwa durch Leistungsdifferenzierungen zustande kommen, ebenso wie ungerechte Gleichheitszustände, welche eher (reform-)politische Fragen berühren. In jedem Fall steht die SA/SP von Anbeginn in engem Zusammenhang mit gesellschaftspolitischen Fragen nach Gleichheit und Gerechtigkeit resp. den kompensatorischen Aufgaben eines Sozialstaates. 
geschieht durch die ,Vermittlung' gesellschaftlicher Ansprüche bzw. Normalitätsvorstellungen und Wertprämissen (etwa hinsichtlich eines menschenwürdigen Lebens) mit subjektiven Bedürfnis- und Lebenslagen. Vermittlung heißt hier: Gesellschafilich als Abweichung definierte, personengebundene Zustände werden dadurch zu korrigieren versucht, dass Personen(-gruppen), denen diese Abweichungen zugeschrieben werden und die daher gesellschaftlich als hilfebedürftig gelten, (Deutungs-)Angebote zur Verfügung gestellt bekommen (Hilfe, Intervention), welche sie befähigen sollen, diesen Zustand aus eigener Krafi (Hilfe zur Selbsthilfe) zu verändern. Die SA/SP erscheint daher häufig als „Normalisierungsarbeit“ (Brunkhorst 1992: 67), weshalb ihr therapeutische, beratende und sozialisatorische Aufgaben (Subjektorientierung) ebenso wie soziale Kontrollfunktionen (Systemorientierung) zufallen. Unschwer lässt sich hierin die oben erörterte Figur des an professionslogischen Kriterien orientierten Handelns (verwissenschaftlichte Krisenbewältigung) unter erschwerten Bedingungen (,doppeltes Mandat') wiedererkennen.

Kernproblematik sozialpädagogischen Handelns ist es daher, eine Balance zwischen sozialer Kontrolle auf der einen und Bedürfnisorientierung resp. Beratung und Therapie auf der anderen Seite aufrechtzuerhalten. Ausgehend von diesem Grunddilemma hat insbesondere Fritz Schütze $(1992,1996$,) auf zentrale und hartnäckige Paradoxien sozialpädagogischen Handelns hingewiesen ${ }^{49}$

\section{Ausblick}

Die professionalisierungstheoretische Debatte ist heute an einem Punkt angelangt, an dem zweierlei unstrittig ist: Zum einen gilt pädagogisches Handeln hinsichtlich seiner Berufsförmigkeit und handlungslogischen Ausrichtung als prinzipiell professionalisierbar. Zum anderen ist der Weg, den ein eigenständiges Konzept pädagogischer Professionalität zu beschreiten hat, markiert: Es geht darum, mithilfe empirischer Untersuchungsstrategien „,das Besondere des pädagogisch-professionellen Handlungsmodus zu rekonstruieren" (Dewe/Ferchhoff/ Radtke 1992: 16). Einschlägige Arbeiten haben gezeigt, dass dieser prinzipiell darin besteht, typische Handlungsparadoxien im Berufsalltag in Interaktion mit Personen(-gruppen) aushalten und ausbalancieren zu müssen, so dass sich die Professionalität pädagogischen Handelns im Kern auf Interaktions- und Kommunikationskompetenzen stützt, welche ein solches Aushalten und -balancieren gewährleisten (sollen). Damit solche Kompetenzen nicht charismatisiert und damit an die ,Genialität' bestimmter Personen (,erzieherisches Talent', ,geborener Erzieher') geknüpft werden, müssen sie als lehr- und lernbare Prozeduren begriffen werden, die Praxis zu reflektieren, um sich dadurch die Möglichkeit zu eröffnen, Praxis rekursiv zu korrigieren.

Im Falle des Lehrerhandelns im Rahmen schulischer Unterrichtskommunikation ließe sich dies dahingehend dynamisieren, dass das Schulsystem aufgrund seiner Tendenz, Ver-

$49 \mathrm{Vgl}$. Schütze (1992: $146 \mathrm{ff}$.). 
antwortung durch Überantwortung an kompensierende Instanzen (SA/SP; Schulsozialarbeit) bzw. durch den Rückzug auf Wissensvermittlung (wodurch Erziehung entweder an die Primär- oder die sog. „Selbstsozialisation“"50 delegiert wird) zu verlagern, Gefahr läuft, einer (latenten) De-Professionalisierung anheim zu fallen. Denn: Schulische Unterrichtskommunikation lässt sich weder dem Gleichaltrigenkollektiv überantworten noch lässt sie sich auf reine Wissensvermittlung verengen, da sie ihre spezifischen Funktionen (Verschränkung von Sozialisations- und Selektionsaufgaben) nur erfüllen kann, indem der Spagat zwischen institutionellem Zwang und klientaler Bedürfnisorientierung aufrechterhalten und immer wieder neu austariert wird. Lehrerhandel ist auf diese Weise immer normativen (Selbst- und Fremd-)Ansprüchen ausgesetzt, d.h. ist notwendigerweise sowohl an Erziehungszielen als auch an der Effektivität ihrer Durchsetzung orientiert bzw. wird daran gemessen. Diese Balancearbeit geschieht immer nur in und durch Kommunikation des Professionellen mit seiner Klientel, sprich durch tagtägliche Interaktion zwischen Lehrer und Schülern im Unterricht. Obwohl das Interaktionssystem ,Lehrer-Schüler' institutionell abgesichert zu sein scheint, muss es kontinuierlich durch das Handeln der Lehrerin interaktional aufrechterhalten werden, zugespitzt formuliert: es muss trotz aller Widersprüchlichkeiten und ,Reibereien“ ,in Gang gehalten ' werden. Insbesondere der Umgang mit ,Sand im Getriebe' (s.o. ,Störungen“) erfordert eine kommunikative Kompetenz, welche professionelles Handeln zunächst dahin gehend anzuleiten hätte, dass (vorschnelle) Verkürzungen (Überantwortungen an die SA/SP resp. an familiale und/oder selbstsozialisatorische Instanzen) vermieden werden. Lehrerprofessionalität - so ließe sich resümieren - basiert (jenseits fachdidaktischer Kompetenzen) zuallererst auf der (kommunikativen) Fähigkeit, Unterricht als soziale Situation interaktiv so zu bewältigen, dass grundlegende Funktionen von Schule gewahrt bleiben (Wissensvermittlung, Selektion, Erziehung). Dies bringt es mit sich, dass neben dem Zur-VerfügungStellen von ,Lernangeboten ' immer auch erzieherisch gehandelt werden muss, d.h. etwa die Relevanz des Wissens zu verdeutlichen, zu motivieren, Lernbarrieren abzubauen, Gefahren des Schulversagens aufzuzeigen, Konsequenzen mangelnden schulischen Engagements zu verdeutlichen etc. Hierzu gehört insbesondere auch der Umgang mit (Schüler-)Aktivitäten, die diese Ziele (absichtlich oder unabsichtlich) konterkarieren, also der Umgang mit Unterrichtsstörungen, da jede Behandlung einer solchen als Testfall gewertet werden kann, ob und in welcher Weise die Lehrperson fähig ist, Ansprüche der Institution mit solchen der Schüler (der Klienten) zu vermitteln. Entscheidend dabei ist „die strategisch geschickte Platzierung von Strukturvorgaben und Struktursicherungsoperationen. Deren Bestandteil kann als wesentlicher Bestandteil professioneller pädagogischer Handlungskompetenz gelten" (Schneider 1997: 219).

Die Frage nach der Lehr- und Lembarkeit einer solchen, genuin pädagogisch-professionellen Kommunikations-/Interaktionskompetenz ${ }^{31}$ führt zu der Forderung, in der Lehrerausbildung - wie der pädagogischen Ausbildung überhaupt - verstärkt interpretativ-fallanalytisch vorzugehen, d.h. anhand von praktischen Fällen (etwa Tonband- oder Videoaufzeichnungen

$50 \mathrm{Vgl}$. Zinnecker 2000 .

51 Innerhalb der Leitthematik einer akademischen Lehrerausbildung geht es hier - wie Ohlhaver/Wernet (1999b) betonen - um den ,spezifisch berufspraktischen Sinn einer wissenschaftlichen Ausbildung jenseits der wissenschaftlichen Aneignung der jeweiligen Unterrichtsfächer" (17). 
aus der Unterrichtspraxis) den Verlauf von Praxis zu reflektieren. ${ }^{52}$ „Denn die Durchführung der Fallanalysen in der Lehrerbildung (...) erlaubt nicht nur eine praxisnahe, problem- und handlungsorientierte Reflexion von Schule und Unterricht, sondern kann dabei auch einen direkten Beitrag zur Verbesserung des Lehrerhandelns im Sinne der Bildung eines berufsadäquaten Habitus leisten“" (Ohlhaver/Wernet 1999b: 15). Die Vermittlung von Theorie (wissenschaftliches Wissen) und Praxis (Handeln im Unterricht) geschieht hier durch Interpretation (vgl. ebenda: 19), d.h. dadurch, dass einerseits mithilfe wissenschaftlichen (methodischen) Wissens die Praxis analysiert und andererseits wissenschaftliches Wissen durch die Interpretation eines Praxisfalls erzeugt wird. Pädagogische Professionalität - verstanden als habituell verdichtete Kommunikationskompetenz - hängt dann in wesentlichen Hinsichten an der Fähigkeit und Bereitschaft, der eigenen Praxis in interpretatorischer Distanz zu begegnen.

\section{Literatur}

Apel, Hans Jürgen et al. (Hrsg.) (1999): Professionalisierung pädagogischer Berufe im historischen Prozess. Bad Heilbrunn: Klinkhardt.

Becker-Mrotzek, Michael/Vogt, Rüdiger (2001): Unterrichtskommunikation. Linguistische Analysemethoden und Forschungsergebnisse. Tübingen: Niemeyer.

Brunkhorst, Hauke (1992): Professionalität, Kollektivitätsorientierung und formale Wertrationalität. Zum Strukturproblem professionellen Handelns aus kommunikationstheoretischer Perspektive. In: Dewe, Bernd/Ferchhoff, Wilfried/Radtke, Frank-Olaf (Hrsg.): Erziehen als Profession. Opladen: Leske + Budrich. 49-69.

Brunkhorst, Hauke (1996): Solidarität unter Fremden. In: Combe, Amo/Helsper, Werner (Hrsg.): Pädagogische Professionalität. Untersuchungen zum Typus pädagogischen Handelns. Frankfurt/M: Suhrkamp. 340-367.

Combe, Arno/Helsper, Werner/Stelmaszyk, Bernhard (Hrsg.) (1999): Forum Qualitative Schulforschung. Bd. 1: Schulentwicklung - Partizipation - Biographie. Weinheim: Deutscher Studien Verlag.

Daheim, Hansjürgen (1992): Zum Stand der Professionssoziologie. Rekonstruktion machttheoretischer Modelle der Profession. In: Dewe, Bernd/Ferchhoff, Wilfried/Radtke, Frank-Olaf (Hrsg.): Erziehen als Profession. Opladen: Leske + Budrich. 21-35.

Dewe, Bernd/Otto, Hans-Uwe (2001): Profession. In: Otto, Hans-Uwe/Thiersch, Hans (Hrsg.): Handbuch Sozialarbeit/Sozialpädagogik. Neuwied; Kriftel: Luchterhand. 1399-1423.

Dewe, Bemd/Ferchhoff, Wilfried/Stüwe, Gerd (1992): Sozialarbeit und Berufswissen. In: Pfaffenberger, Hans/ Schenk, Manfred (Hrsg.): Sozialarbeit zwischen Berufung und Beruf. Professionalisierungs- und Verwissenschaftlichungsprobleme der SozialarbeivSozialpädagogik. Münster; Hamburg: Lit Verlag. 189-204.

Dewe, Uwe/Ferchhoff, Wilfried/Radtke, Frank-Olaf (1992): Auf dem Wege zu einer aufgabenzentrierten Professionstheorie pädagogischen Handelns. In: Dewe, Bernd/Ferchhoff, Wilfried/Radtke, Frank-Olaf (Hrsg.): Erziehen als Profession. Opladen: Leske + Budrich. 7-20.

Ferchhoff, Wilfried (1993): Was können und wissen Sozialpädagogen? Neue professionstheoretische Überlegungen zum Theorie - Praxis - Verhältnis in der Sozialpädagogik. In: Pädagogische Rundschau 6. 47. 705-719.

Fischer, Aloys (1950) [1921]: Erziehung als Beruf. In: Ders.: Leben und Werk. Bd. 2. hrsg. v. K. Kreitmair. Munchen. 31-71.

Giesecke, Hermann (1996): Das „Ende der Erziehung“. Ende oder Anfang pädagogischer Professionalisierung. In: Combe, Amo/Helsper, Werner (Hrsg.): Pädagogische Professionalität. Untersuchungen zum Typus pädagogischen Handelns. FrankfurtM: Suhrkamp. 391-403.

Harrach, Eva-Marie von/Loer, Thomas/Schmidtke, Oliver (2002): Verwaltung des Sozialen: Formen der subjektiven Bewältigung eines Strukturkonflikts. Konstanz: UVK.

$52 \mathrm{Vgl}$. insbesondere Ohlhaver/Wernet (1999b). 
Hartmann, Heinz (1972): Arbeit, Beruf, Profession. In: Luckmann, Thomas/Sprondel, Walter Michael (Hrsg.): Berufssoziologie. Köln: Kiepenheuer \& Witsch. 36-52.

Helsper, Werner (1996): Antinomien des Lehrerhandelns in modemisierten pädagogischen Kulturen. Paradoxe Verwendungsweisen von Autonomie und Selbstverantwortlichkeit. In: Combe, Amo/Helsper, Werner (Hrsg.): Pädagogische Professionalităt. Untersuchungen zum Typus pädagogischen Handelns. FrankfurtM: Suhrkamp. 521-569.

Krummheuer, Götz/Naujok, Natalie (1999): Grundlagen und Beispiele Interpretativer Unterrichtsforschung. Opladen: Leske + Budrich

Kurtz, Thomas (2002): Berufssoziologie. Bielefeld: Transcript-Verlag.

Luhmann. Niklas (1969): Funktionen und Folgen formaler Organisation. Berlin: Duncker \& Humblot.

Luhmann, Niklas (1995): Das Kind als Medium der Erziehung. In: Ders.: Soziologische Aufklärung, Bd. 6: Die Soziologie und der Mensch. Opladen: Leske + Budrich. 204-228.

Luhmann, Niklas/Schorr, Karl Eberhard (Hrsg.) (1982): Zwischen Technologie und Selbstreferenz. Fragen an die Pädagogik. Frankfurc/M: Suhrkamp. 11-40.

Merten, Roland/Olk. Thomas (1996): Sozialpädagogik als Profession. Historische Entwicklung und künftige Perspektiven. In: Combe. Arno/Helsper, Werner (Hrsg.): Pädagogische Professionalität. Untersuchungen zum Typus pädagogischen Handelns. Frankfurt/M: Suhrkamp. 570-613

Münchmeier, Richard (1980): „Wofür wirst du eigentlich bezahlt?“ Zur Professionalisierung in der Jugendarbeit. In: Böhnisch, Lothar/Münchmeier, Richard/Sander, Ekkehard (Hrsg.): Abhauen oder Bleiben? Berichte und Analysen aus der Jugendarbeit. München: Piper. 117-131.

Nolda, Sigrid (2000): Interaktion in pädagogischen Institutionen. Opladen: Leske + Budrich.

Oevermann, Ulrich (1996): Skizze einer revidienten Theorie professionalisierten Handelns. In: Combe, Amo/Helsper, Werner (Hrsg.): Pädagogische Professionalität. Untersuchungen zum Typus pädagogischen Handelns. Frankfurt/M.: Suhrkamp. 70-182.

Ohlhaver, Frank/Wernet, Andreas (1999a): Schulforschung - Fallanalyse - Lehrerbildung. Diskussionen am Fall. Opladen: Leske + Budrich

Ohlhaver, Frank/Wernet, Andreas (1999b): Zwischen Pädagogik und Erziehungswissenschaft: Ansätze zur systematischen Begrundung eines interpretativ-fallanalytischen Vorgehens in der Lehrerbildung. In: Dies. (Hrsg.): Schulforschung, Fallanalyse, Lehrerbildung. Diskussionen am Fall. Opladen: Leske + Budrich. 11-28.

Parsons, Talcott (1965) [engl.: 1951]: Struktur und Funktion der modernen Medizin. Eine soziologische Analyse. In: König, René/Tönnesmann, Margret (Hrsg.): Probleme der Medizinsoziologie, Sonderheft 3 der Kölner Zeitschrift für Soziologie und Sozialpsychologie. 10-57.

Pfadenhauer, Michaela (2003): Professionalisierung. Eine wissenssoziologische Rekonstruktion professioneller Kompetenzdarstellungskompetenz. Opladen: Leske + Budrich.

Schneider, Wolfgang L. (1997): Die Analyse von Struktursicherungsoperationen als Kooperationsfeld von Konversationsanalyse, objektiver Hermeneutik und Systemtheorie. In: Sutter, Tilmann (Hrsg.): Beobachtung verstehen - Verstehen beobachten. Perspektiven einer konstruktivistischen Hermeneutik. Opladen: Westdeutscher Verlag. 164-227.

Schutze, Fritz (1992): Sozialarbeit als ,bescheidene' Profession. In: Dewe, Uwe/Ferchhoff, Wilfried/Radtke, FrankOlaf (Hrsg.): Erziehen als Profession. Opladen: Leske + Budrich. 132-170.

Schütze, Fritz (1996): Organisationszwänge und hoheitsstaatliche Rahmenbedingungen im Sozialwesen: Ihre Auswirkung auf die Paradoxien des professionellen Handelns. In: Combe, Amo/Helsper, Werner (Hrsg.): Pädagogische Professionalität. Untersuchungen zum Typus pädagogischen Handelns. Frankfur/M: Suhrkamp. 183275.

Stichweh, Rudolf (1992): Professionalisierung, Ausdifferenzierung von Funktionssystemen, Inklusion. Betrachtungen aus systemtheoretischer Sicht. In: Dewe, Uwe/Ferchhoff, Wilfried/Radtke, Frank-Olaf (Hrsg.): Erziehen als Profession. Opladen: Leske + Budrich. 36-48.

Stichweh, Rudolf (1996): Professionen in einer funktional differenzierten Gesellschaft. In: Combe, Arno/Helsper, Werner (Hrsg.): Pädagogische Professionalität. Untersuchungen zum Typus pädagogischen Handelns. Frankfurt/M: Suhrkamp. 49-69.

Wernet, Andreas (2003): Pädagogische Permissivität. Schulische Sozialisation und pädagogisches Handeln jenseits der Professionalisierungsfrage. Opladen: Leske + Budrich

Zinnecker, Jürgen (2000): Selbstsozialisation - Essay über ein aktuelles Konzept. In: Zeitschrift für Sozialisation und Erziehungssoziologie, 3. 272-290. 


\section{Einführende Literatur}

Combe, Amo/Helsper, Werner (Hrsg.) (1996): Pädagogische Professionalität. Untersuchungen zum Typus pädagogischen Handelns. Frankfur//M: Suhrkamp.

Dewe, Uwe/Ferchhoff, Wilfried/Radtke, Frank-Olaf (Hrsg.) (1992): Erziehen als Profession. Opladen: Leske + Budrich.

Dewe, Bernd/Ferchhoff, Wilfried/Scherr, Albert/Stüwe, Gerd (2001): Professionelles soziales Handeln. Soziale Arbeit im Spannungsfeld zwischen Theorie und Praxis. Weinheim; München: Juventa.

Giesecke, Hermann (2000): Pädagogik als Beruf. Grundformen pädagogischen Handelns. Weinheim; München: Juventa.

\section{Weiterführende Literatur}

Bastian, Johannes/Helsper, Werner/Reh, Sabine/Schelle, Carla (Hrsg.) (2000): Professionalisierung im Lehrerberuf. Von der Kritik der Lehrerrolle zur pädagogischen Professionalität. Opladen: Leske + Budrich.

Pfadenhauer, Michaela (2003): Professionalisierung. Eine wissenssoziologische Rekonstruktion professioneller Kompetenzdarstellungskompetenz. Opladen: Leske + Budrich

Ohlhaver, Frank/Wernet, Andreas (1999): Schulforschung - Fallanalyse - Lehrerbildung. Diskussionen am Fall. Opladen: Leske + Budrich.

Wernet, Andreas (2003): Pädagogische Permissivität. Schulische Sozialisation und pädagogisches Handeln jenseits der Professionalisierungsfrage. Opladen: Leske + Budrich. 